\title{
Identification of plastic constitutive parameters at large deformations from three dimensional displacement fields
}

\author{
Marco Rossi • Fabrice Pierron
}

Received: date / Accepted: date

\begin{abstract}
The aim of this paper is to provide a general procedure to extract the constitutive parameters of a plasticity model starting from displacement measurements and using the Virtual Fields Method. This is a classical inverse problem which has been already investigated in the literature, however several new features are developed here. First of all the procedure applies to a general three-dimensional displacement field which leads to large plastic deformations, no assumptions are made such as plane stress or plane strain although only pressure-independent plasticity is considered. Moreover the equilibrium equation is written in terms of the deviatoric stress tensor that can be directly computed from the strain field without iterations. Thanks to this, the identification routine is much faster compared to other inverse methods such as finite element updating. The proposed method can be a valid tool to study complex phenomena which involve severe plastic deformation and where the state of stress is completely triaxial, e.g. strain localization or necking occurrence. The procedure has been validated using a three dimensional displacement field obtained from a simulated experiment. The main potentialities as well as a first sensitivity study on the influence of measurement errors are illustrated.
\end{abstract}

M. Rossi - F.Pierron

LMPF, Arts et Métiers ParisTech, Rue St Dominique, B.P. 508, 51006 Châlons-en-Champagne, France

E-mail: marco.rossi@ensam.eu

Tel.: +33 (0)326 699176

Fax: +33(0)326699176

F. Pierron

E-mail: Fabrice.Pierron@ensam.eu
Keywords Constitutive modelling · Inelastic and finite deformation - Inverse methods · Numerical algorithms

\section{Introduction}

The characterization of the plastic behaviour of metals is commonly obtained by means of uniaxial tensile tests. The main limitation of such tests is the occurrence of plastic instabilities, e.g. diffuse and localized necking, as the plastic deformation increases. The necking makes the stress state inside the specimen triaxial and leads to fracture $[1,2,3]$. Moreover extensive research in the field of plasticity and the new materials continuously developed in this area led to new constitutive models that often require the identification of a great number of parameters. This area of research is extremely wide, examples of recent models developed in anisotropic plasticity can be found in Cazacu et al. [4], Plunkett et al. [5] and Barlat et al. [6] which extend the criteria of Barlat et al. [7] and Karafillis and Boyce [8], for instance. Other interesting theories can be found in Darrieulat and Piot [9], Feigenbaum and Dafalias [10], Voyiadjis et al. [11]. Vegter and van den Boogaard [12] use a piecewise description of the anisotropic yield limit using Bézier splines, Bai and Wierzbicki [13] introduce the dependence on the third stress invariant. Many other authors could be cited here, however it is evident that there is an increasing interest in the field, also from the industrial side, since the new models can be implemented in FE codes, leading to a more accurate de- 


\begin{tabular}{|c|c|c|c|}
\hline Variables & & $\mathbf{U}, \mathbf{V}$ & right and left stretch tensors \\
\hline $\mathbf{a}$ & acceleration & & displacement vector \\
\hline b & specific body force & {$\left[\mathbf{u}_{k}^{N}\right]$} & matrix of the nodal displacement at \\
\hline$\left[\mathbf{B}_{k}\right]$ & $\begin{array}{l}\text { matrix to evaluate the gradient at the } \\
\text { integration point of element } k\end{array}$ & & $\begin{array}{l}\text { element } k \\
\text { virtual velocity vector }\end{array}$ \\
\hline $\begin{array}{l}\mathscr{B}_{0}, \mathscr{B}_{t} \\
d a_{0}, d a\end{array}$ & $\begin{array}{l}\text { body in the reference and current placement } \\
\text { element of area in the reference and }\end{array}$ & {$\left[\delta \mathbf{v}_{k}^{N}\right]$} & $\begin{array}{l}\text { matrix of the nodal virtual velocity at } \\
\text { element } k\end{array}$ \\
\hline$d m_{0}, d m$ & $\begin{array}{l}\text { current placement } \\
\text { element of mass in the reference and }\end{array}$ & $\mathbf{x}_{0}, \mathbf{x}$ & $\begin{array}{l}\text { position vector in the reference and } \\
\text { current placement }\end{array}$ \\
\hline & current placement & $\nu$ & Poisson's ratio \\
\hline$d v_{0}, d v$ & $\begin{array}{l}\text { element of volume in the reference and } \\
\text { current placement }\end{array}$ & $\begin{array}{l}\boldsymbol{\xi}=\left\{X_{i}\right\} \\
\sigma_{T}\end{array}$ & $\begin{array}{l}\text { constitutive parameters vector } \\
\text { equivalent stress }\end{array}$ \\
\hline$E$ & Young's modulus & $\sigma_{Y}$ & yield stress \\
\hline $\mathbf{E}=\ln \mathbf{V}$ & spatial logarithmic strain tensor & $\Phi_{p}$ & yield function \\
\hline $\mathbf{E}^{p \bullet}$ & plastic strain rate & $\chi$ & motion function \\
\hline $\begin{array}{l}\Delta \mathbf{E}_{k}^{p} \\
F\end{array}$ & $\begin{array}{l}\text { plastic strain increment at element } k \\
\text { total traction force in the test }\end{array}$ & Numbering & \\
\hline $\mathbf{F}$ & deformation gradient & $k$ & index referring to the element \\
\hline$\delta \mathbf{F}^{\bullet}$ & virtual velocity gradient & $N_{c p}$ & number of constitutive parameters \\
\hline f & resultant of the external forces & $N_{E}$ & number of elements \\
\hline$\delta \mathbf{D}$ & virtual rate of deformation tensor & $N_{t}$ & number of time steps \\
\hline I & unit tensor & $t$ & index referring the time step \\
\hline$\widehat{\mathbf{N}}_{P}{ }_{k}^{(t)}=\left\{\widehat{n}_{i j}^{P}\right\}$ & normalised tensor of the plastic flow & & \\
\hline$\widehat{\mathbf{N}}_{S}{ }_{k}^{(t)}=\left\{\widehat{n}_{i j}^{S}\right\}$ & normalised tensor of the deviatoric stress & Operators & \\
\hline $\mathbf{n}_{0}, \mathbf{n}$ & $\begin{array}{l}\text { normal vector in the reference and } \\
\text { current placement }\end{array}$ & | | & $\begin{array}{l}\text { inner or scalar product } \\
\text { modulus of a tensor or }\end{array}$ \\
\hline$p$ & equivalent cumulated plastic strain & & absolute value of a scalar \\
\hline $\mathbf{R}$ & rotation tensor & Grad,grad & material and spatial gradient operator \\
\hline$R$ & Lankford parameter & & \\
\hline $\mathbf{t}$ & surface load & Generic not & ations \\
\hline $\mathbf{S}=\left\{s_{i j}\right\}$ & deviatoric part of the Cauchy stress tensor & scalars & italic letters like $A, B, a, b, \alpha, \beta$. \\
\hline $\mathbf{T}=\left\{\sigma_{i j}\right\}$ & Cauchy stress tensor & vectors & small letters in bold like $\mathbf{a}, \mathbf{b}, \boldsymbol{\alpha}, \boldsymbol{\beta} \ldots$ \\
\hline $\mathbf{T}^{1 P K}$ & 1st Piola-Kirchhoff stress tensor & tensors & large Latin letters in bold like $\mathbf{A}, \mathbf{B}$, . \\
\hline
\end{tabular}

scription of the manufacturing processes which involve severe plastic deformation.

Often the main limitation to the applicability of a new model resides in the difficulty of experimentally identifying the required constitutive parameters. Indeed, in order to characterize the plastic behaviour of such complex materials up to large deformation the standard uniaxial tests become inadequate.

More sophisticated experimental procedures can be employed, for instance hydraulic bulge tests $[15,16]$, bending tests $[17,18]$, torsion test, punch test, biaxial test [19], combined tests [20,21] etc.

In general, in these tests, it is not possible to identify directly the parameters of the constitutive equation because the stress state in the specimen is not uniform during the deformation process and it cannot be computed a priori from the specimen geometry. An inverse approach which involves finite element (FE) updating

\footnotetext{
1 The notation used in the paper follows the indications given in Elasticity and Plasticity of Large Deformation (Bertram, 2008) [14].
}

is often adopted $[22,23,24,25,26]$, i.e. a FE model of the test is built up and the constitutive parameters of the plasticity model are iteratively modified in order to achieve the best agreement with the quantities measured in the actual tests. The comparison can be made either on the force measured during the test $[27,28]$ or including also the displacement measured at the surface of the specimen, for instance using full-field measurements $[22,26]$. This procedure can become highly time-consuming, especially if the constitutive model or the geometry are complex, because at each iteration a complete FE computation has to be performed. Another drawback is that often the actual boundary conditions are not perfectly reproduced in the FE model, introducing errors in the subsequent identification.

Other methods have been proposed to directly evaluate the constitutive equations from the displacement and strain field measured by an optical full-field technique, examples of applications in plasticity are given in Rossi et al. [29] and Latourte et al. [30]. An interesting approach is the one proposed by Romano et 
al. [31] where the identification procedure only involves displacement measurements, the method was used by the same authors to identify the material parameters using MRI-based displacement measurements [32]. A complete overview of the identification methods based on full-field measurement is given by Avril et al. [33]

Among such methods the Virtual Fields Method (VFM) is one of the most used and well-known. The advantage of using full-field measurements in the identification process is that a lot of information can be obtained from a single test, especially if it is designed in such a way as to produce a suitable heterogeneous strain field. A review of the VFM is given by Grédiac et al. [34] and its application to elasto-plasticity is described in Grédiac and Pierron [35] and Avril et al. [36], moreover Pierron et al. [37] extended the method to cyclic loads and kinematic hardening. In dynamic, examples of application of the VFM are given in Pierron et al. [38] and Avril et al. [39].

In the aforementioned papers the VFM was applied to isotropic plasticity at small strain, here it is extended to large plastic deformation and an anisotropic behaviour is considered. The main features introduced are:

- the proposed procedure is developed for a general three-dimensional displacement field, no conditions such as plane stress or plane strain are imposed;

- dealing with large plastic deformation, the plastic strain is assimilated to the total one and the elastic part of the deformation is neglected, in such way the elastic properties do not enter in the identification of the plastic constitutive parameters. Besides, using the total deformation, the procedure is less sensitive to the measurement noise since the magnitude of the strains to be measured is usually large compared to the sensitivity of the measurement system;

- at each step the equilibrium equation is written in terms of the deviatoric stress tensor which can be directly evaluated from the displacement field, thus no iterations are required even if a non linear hardening law is used. This feature makes the identification procedure very fast even when a great amount of data have to be processed and a large number of parameters have to be identified.

All these aspects will be studied in depth in the following. Since the proposed method can be adapted to various plasticity models, the intent here is also to provide a framework to the reader which allows to use the same procedure with different plasticity models.

In this study the Hill48 anisotropic yield criterion was used and the identification routine was validated using simulated data from a tensile test on a notched specimen where significant strain localization is produced. The use of simulated data enables to have a direct evaluation of the correctness of the identified parameters. Subsequent papers will be dedicated to real experiments.

On this subject it can be said that the possibility of experimentally measuring a volume displacement field during a mechanical test has been demonstrated in several studies. For example, the X-ray tomography plus digital volume correlation (DVC) can be successfully used to obtain volume full-field experimental results $[40,41,42,43]$. The DVC requires a random internal pattern which can be inherent to the material microstructure, as in bones or foams, or introduced artificially [44, $45,46]$. However, other techniques can be adopted too, for instance, in sheet metals, the volume displacement field inside the necking region can be reconstructed starting from surface measurement on both faces [47]. The present approach could be also adapted to large deformation on biological tissues with MRI or MRE [48]. Although the development of volume full-field measurement techniques is still in its infancy compared to the surface ones, the technologies are rapidly progressing. We believe that it is worth putting efforts in identification procedures which involve three-dimensional data since many complex phenomena such as plastic instabilities, necking, strain localization can be entirely detailed only resorting to a three-dimensional description.

\section{Simulated tensile test}

The aim of the proposed method is the identification of the plastic constitutive parameters starting from the displacement field measured during a test; this is often referred to as an inverse problem. The known quantities are the geometry of the specimen, the measured displacement field at each step of the test, the loading conditions, plus the plasticity model which is assumed a priori. The unknowns are the constitutive parameters corresponding to the chosen plasticity model.

Usually the displacement is measured with a fullfield optical technique, in the present case the test has been simulated using an FE model. The purpose of this paper is indeed to validate the identification procedure and this can be easily done using simulated tests where the parameters to be identified are the ones input in the simulations.

The FE model does not enter in the identification procedure and formally there are no differences in using actual data or simulated data. Of course simulated data are not affected by measurement errors and the displacement field is consistent with the material constitutive model input into the FEM. 


\subsection{FE model of the test}

The simulated test is a tensile test on a thick notched shaped specimen, the geometry is illustrated in Fig. 1. The specimen is $4 \mathrm{~mm}$ thick and is made of an aluminum-like material which will be described in the following. The displacement field used as input in the identification procedure is taken at the centre of the specimen in the zone named volume of measurement.

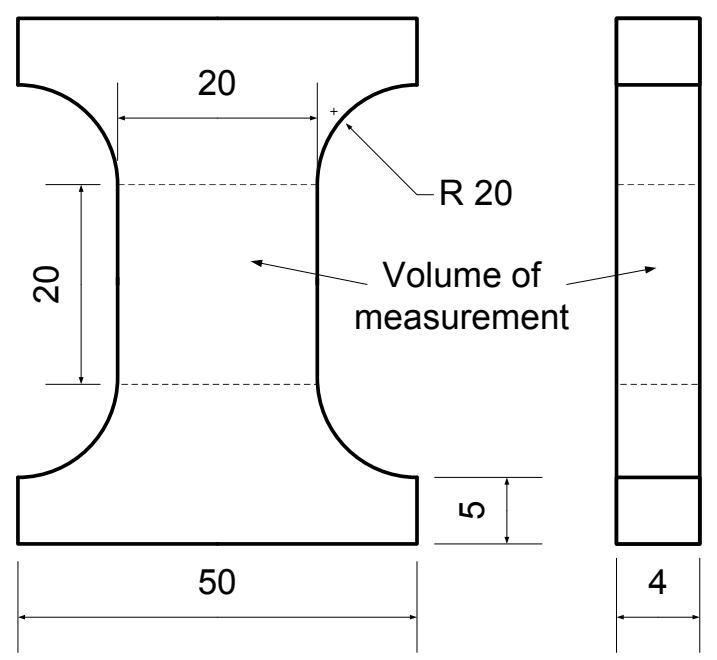

Fig. 1 Geometry of the specimen used in the simulated test (dimensions in $\mathrm{mm}$ ). The three dimensional displacement field used in the identification procedure is the one inside the volume of measurement.

The peculiarity of such a test is that, although the shape of the central zone is rectangular, because of the notch radii at the sides, the strain rapidly localizes at the centre of the specimen leading to a heterogeneous and triaxial stress-strain field.

ABAQUS/Standard has been used to create the numerical model. The adopted mesh is illustrated in Fig. 2, 8-node brick elements have been employed to build up the model. Thanks to the geometrical symmetries of the specimen, only $1 / 8$ of the specimen has to be used in the computation. A global Cartesian coordinate system is defined with the following orientations: 1-axis along the width of the specimen, 2-axis along the traction direction, 3-axis along the thickness, the origin is positioned at the centre of the specimen.

The chosen plasticity model is the classical Hill48 criterion for anisotropic plasticity [49] with the associated flow rule. The equivalent stress, expressed in terms of the deviatoric stress, writes:

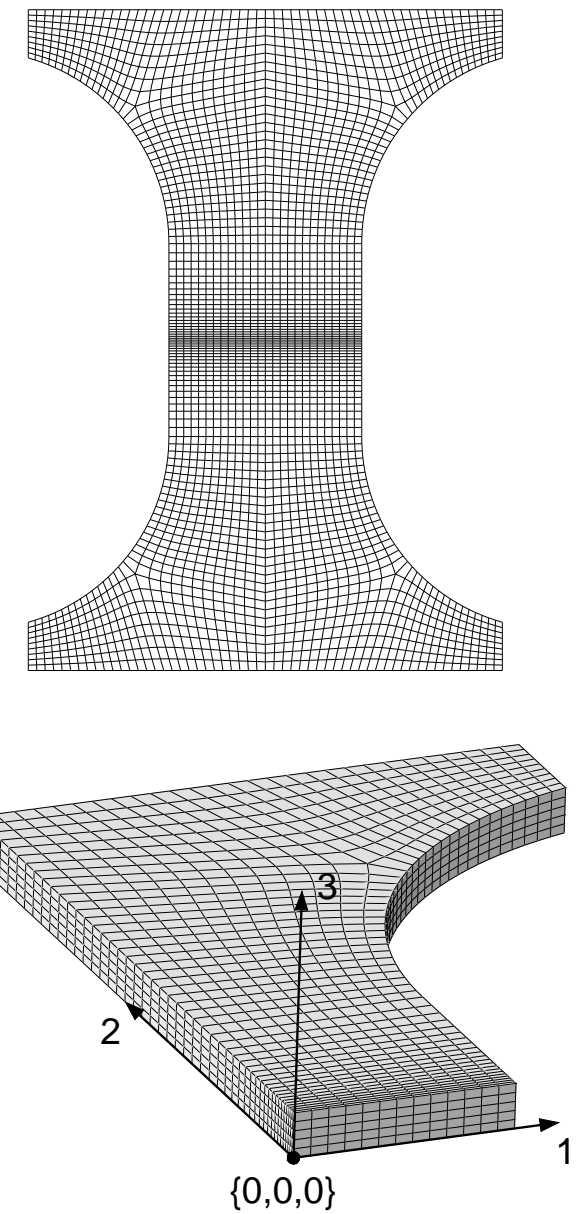

Fig. 2 Mesh of the FE model and used global coordinate system. Only $1 / 8$ of the specimen can be used in the computation thanks to the symmetries along 3 planes.

$$
\begin{aligned}
& \sigma_{T}(\mathbf{S})=\left[f\left(s_{22}-s_{33}\right)^{2}+g\left(s_{33}-s_{11}\right)^{2}+\right. \\
& \left.h\left(s_{11}-s_{22}\right)^{2}+l s_{23}{ }^{2}+m s_{31}{ }^{2}+n s_{12}{ }^{2}\right]^{\frac{1}{2}}
\end{aligned}
$$

where $f, g, h, l, m, n$ are constants which describe the anisotropic behaviour. Assuming that only normal anisotropy is present, the six parameters can be rewritten as a function of the parameter $R$, introduced by Lankford et al. [50], which represents the ratio of the transverse strain to the through thickness strain in a uniaxial tensile test [51], viz:

$$
\begin{aligned}
& f=\frac{1}{1+R} ; g=\frac{1}{1+R} ; h=\frac{R}{1+R} ; \\
& l=\frac{3}{2} ; m=\frac{3}{2} ; n=\frac{1+2 R}{1+R} ;
\end{aligned}
$$

The hardening behaviour is described using an isotropic hardening model and a modified power law to 


\begin{tabular}{ll}
\hline \multicolumn{2}{l}{ Elastic properties } \\
\hline Young's Modulus & $70 \mathrm{GPa}$ \\
Poisson's ratio & 0.3 \\
\hline \multicolumn{2}{l}{ Constitutive parameters of the plasticity model } \\
\hline$K_{H}$ & $310 \mathrm{MPa}$ \\
$\varepsilon_{0}$ & 0.02 \\
$N_{H}$ & 0.08 \\
$R$ & 0.6
\end{tabular}

Table 1 Material properties input in the FE model to generate simulated experiments.

compute the relation between the yield stress and the equivalent plastic strain, i.e.:

$\sigma_{Y}=K_{H}\left(p+\varepsilon_{0}\right)^{N_{H}}$

where $\sigma_{Y}$ is the yield stress, $p$ is the equivalent plastic strain and $K_{H}, \varepsilon_{0}$ and $N_{H}$ are parameters. The values of the parameters used in the simulated test are reported in Table 1, they will be the reference constitutive parameters to be identified in the inverse problem.

In Fig. 3 a rendering of the deformed shape of the specimen is shown as well as a cut view of the equivalent plastic strain field produced inside the necking zone. At the end of the test the maximum level of plastic deformation at the centre is around 1.

The specimen is loaded with a linearly increasing displacement applied to the end nodes, simulating a tensile test performed in displacement control. The maximum displacement is $4.6 \mathrm{~mm}$, the test is subdivided in 50 equispaced steps. A reference time $t$ is introduced with $t=0$ at the beginning and $t=1$ at the last step.

In Fig. 4 the obtained force vs displacement curve is plotted, in the same graph three time steps at different stages of the test are highlighted, for these steps the obtained equivalent plastic strain maps are then shown in Fig. 5. The strain maps are taken at the top surface of the specimen and at the mid plane in order to describe the plastic deformation inside and outside the specimen. The plastic strain distribution changes during the test and localizes towards the centre. It can be observed that, in the last part of the test, the plastic strain obtained inside the specimen is around $25 \%$ greater than the one at the surface. It is clear that, in such a kind of problems with strain localization, volume strain measurements are necessary to be able to correctly describe the problem.

Finally, in Fig. 6 the stress field obtained inside the specimen at the end of the test is shown. The state of stress is triaxial, all the components in the three directions are consistently different from zero and the plane stress assumption is not valid anymore.
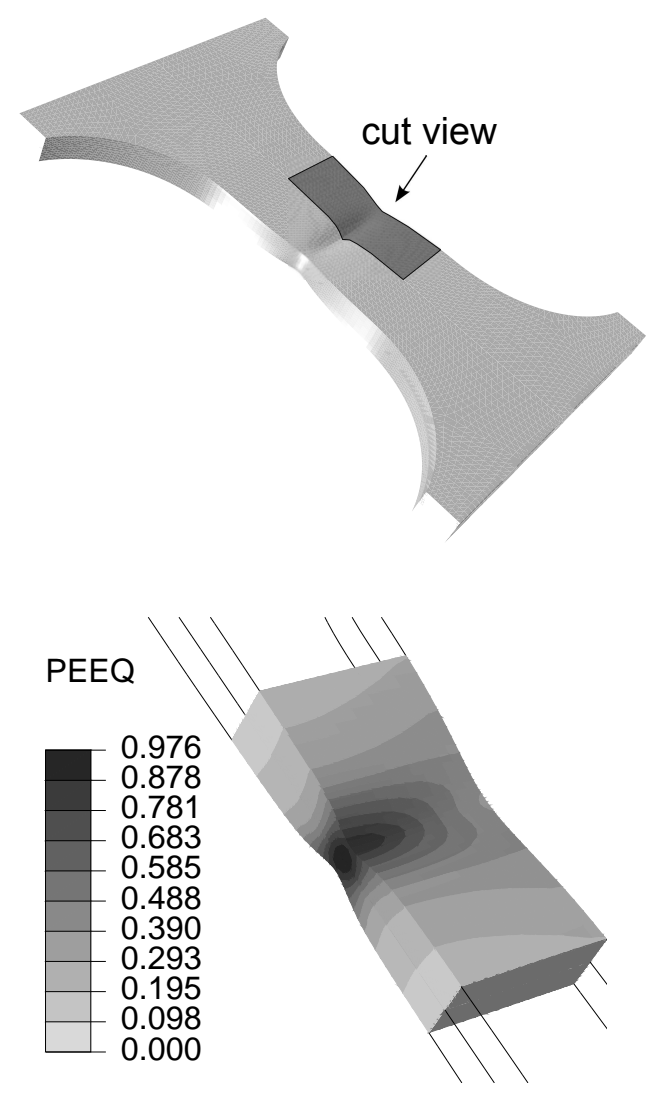

Fig. 3 Numerical simulation of the test. A localized necking is produced at the centre of the specimen. In the cut view on the right the equivalent plastic strain field (PEEQ in ABAQUS/Viewer) inside the specimen is illustrated. The maximum value of the plastic strain is reached at the centre.

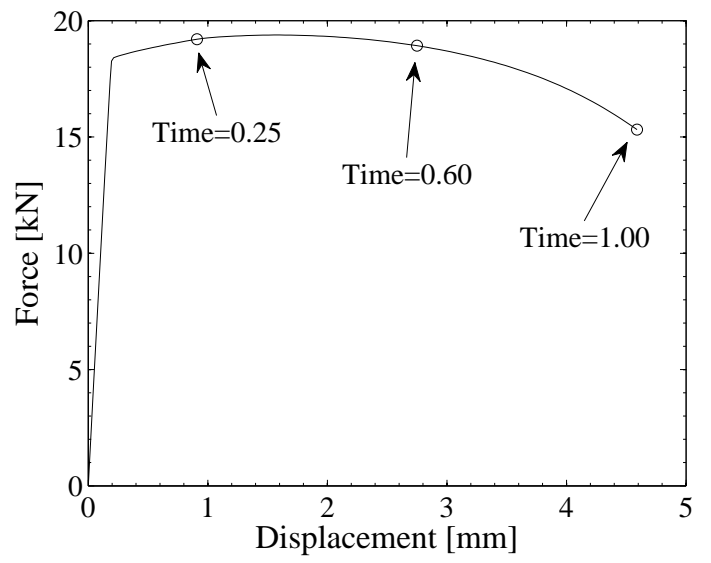

Fig. 4 Force vs displacement curve obtained from the simulated tests. The maximum imposed displacement is $4.6 \mathrm{~mm}$ which correspond to the reference time $t=1.00$. 
Time $=\mathbf{0} .25$

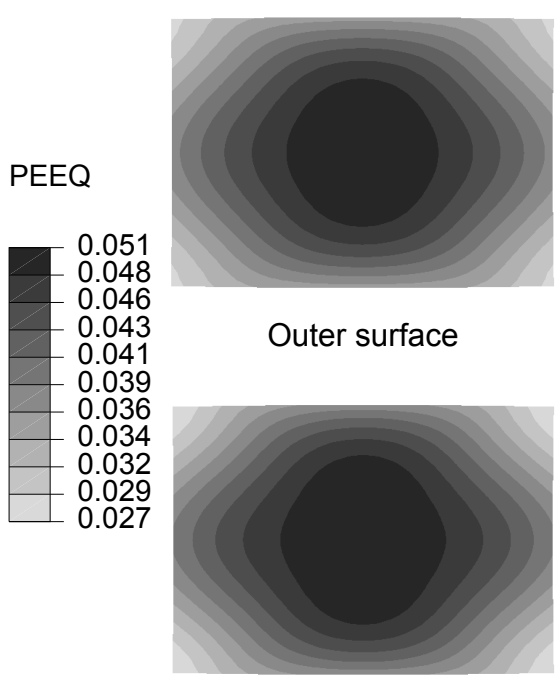

Mid surface
Time $=\mathbf{0 . 6 0}$

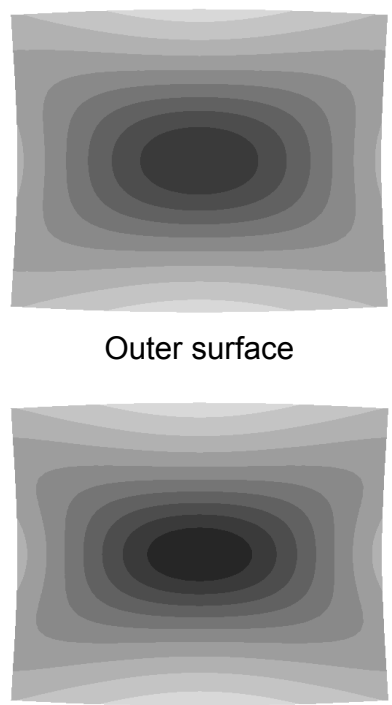

Mid surface
Time $=1.00$

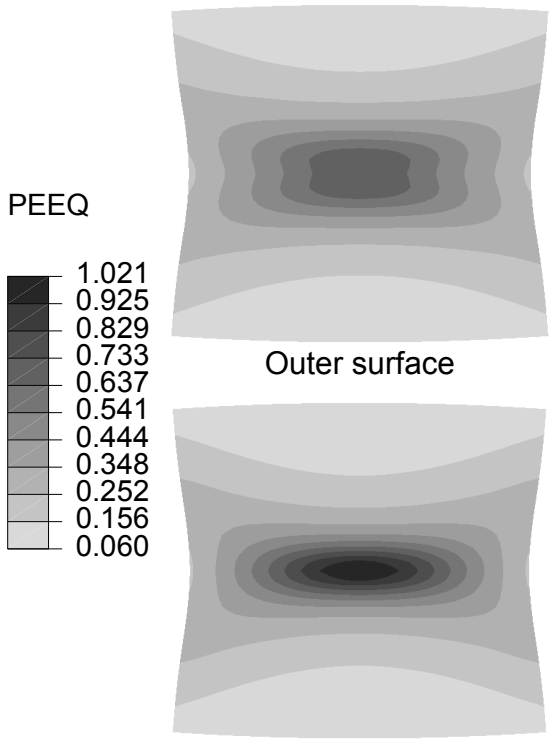

Mid surface

Fig. 5 Equivalent plastic strain maps obtained at different time steps of the test. For each time step two maps are represented, one taken at the outer surface of the specimen and the other in the mid plane inside the specimen.
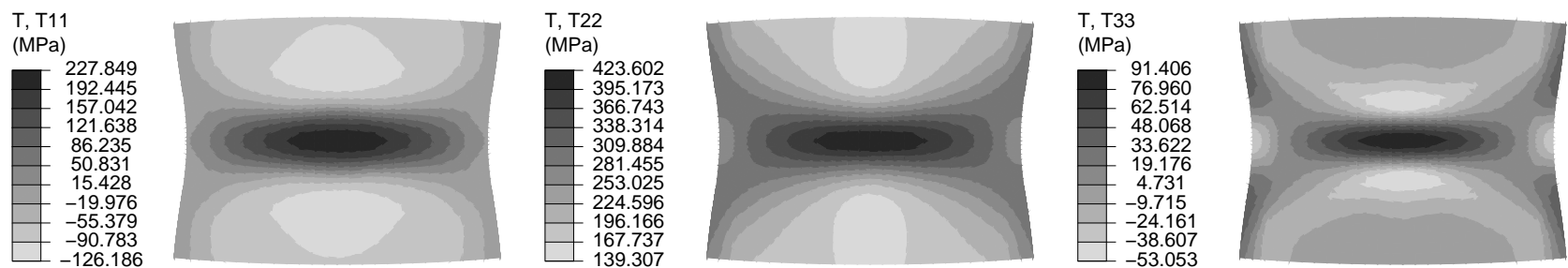

Fig. 6 Components of the stress tensor in the three directions at the mid plane of the specimen at $t=1.00$. The produced state of stress is completely triaxial.

\subsection{Extraction of the simulated displacement field}

The displacement field is given in terms of the nodal displacement $\mathbf{u}^{N}$ of a 3D mesh over the volume of measurement. This is a convenient way of expressing the measured or simulated data, indeed the displacement at each point inside the volume of measurement can be obtained as a function of the nodal displacement using the element shape functions.

The mesh adopted to describe the displacement field can be independent from the mesh adopted in the FE model. The procedure to extract the simulated displacement field is explicated in Fig. 7. The FEM model is used to obtain the displacement field in the specimen during the deformation process, then the displacement field in the volume of measurement is obtained as a linear interpolation over a measurement mesh which can be selected by the user. In practise this procedure can be used to smooth the data and reduce its size [52].
In order to check the dependency of the identification routine on the adopted measurement mesh, four different measurement meshes have been taken into consideration, named Mesh-0, Mesh-1, Mesh-2 and Mesh-3. Mesh-0 is the one adopted for the FE model (cf. Fig. 2), in the volume of measurement there are $26 \times 42 \times 10$ elements and there is a refinement in the 2-direction towards the centre of the specimen where the strain localizes. Mesh-1 has the same number of elements as the previous one but there is no refinement in the 2 direction. Mesh-2 is a coarser mesh with $20 \times 20 \times 6$ elements. Finally, Mesh-3 is a very coarse mesh with $8 \times 8 \times 4$ elements.

In conclusion the inverse problem to be solved has been summarized in Table 2, the known quantities are obtained from the simulated test, the unknown parameters are then identified with the proposed procedure and compared with the one used as input in the simulated test. 


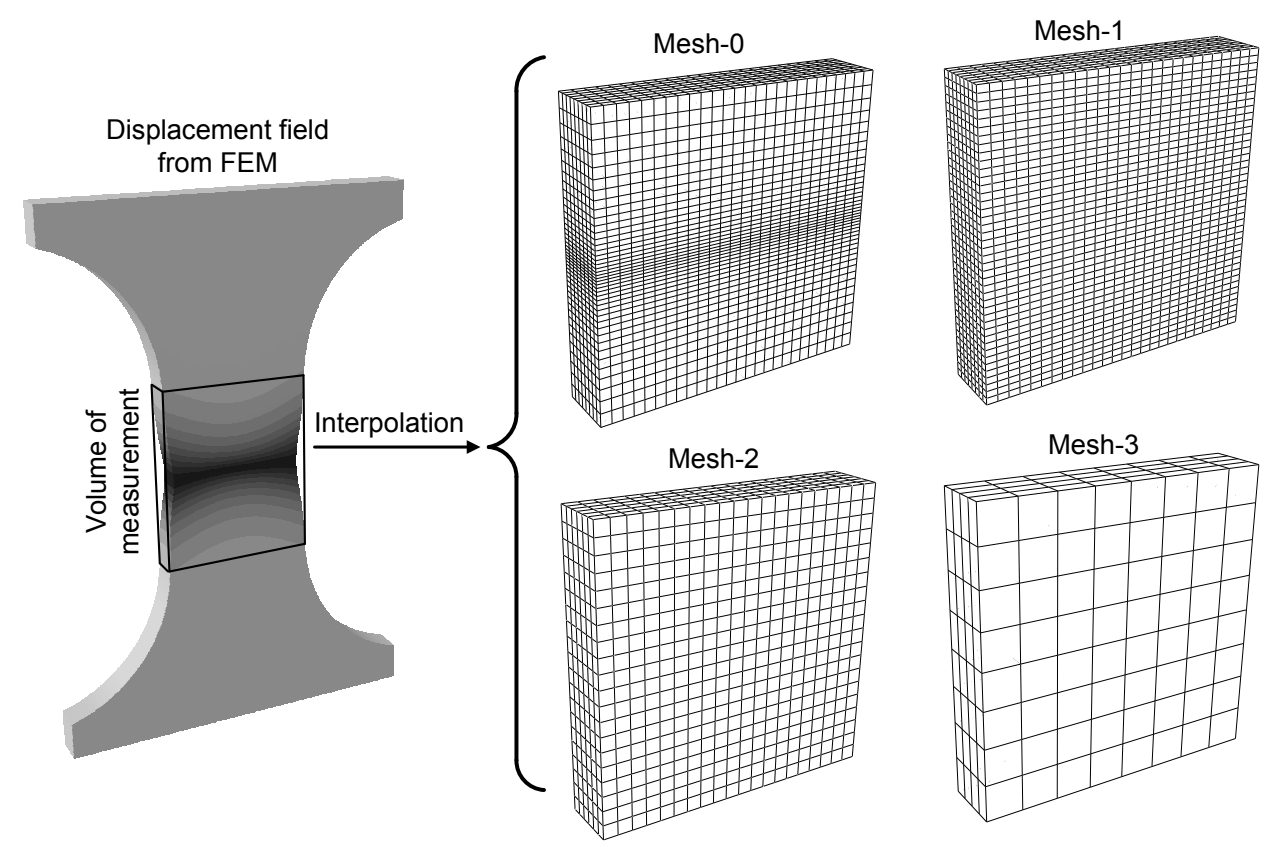

Fig. 7 The displacement field is extracted from the FE model by linear interpolation of the nodal value over a 3D measurement mesh. The size of the mesh adopted to describe the displacement field can be decided independently by the user.

\begin{tabular}{ll}
\hline Known & Unknown \\
\hline Geometry & Constitutive parameters \\
Traction force $(F)$ & $\boldsymbol{\xi}=\left\{R, K_{H}, \varepsilon_{0}, N_{H}\right\}$ \\
Displacement field $\left(\mathbf{u}^{N}\right)$ & \\
Plasticity model & \\
$\quad$ Yield criterion: Hill48 & \\
Flow rule: associative flow rule & \\
Hardening law: $\sigma_{Y}=K_{H}\left(p+\varepsilon_{0}\right)^{N_{H}}$ & \\
\hline
\end{tabular}

Table 2 Known and unknown parameters in the inverse problem.

\section{Identification procedure}

The virtual fields method is based on the principle of virtual power and allows to retrieve the constitutive parameters from a set of strain fields measured over a specimen during a test. An application of the VFM to elasto-plasticity has already been presented in Grédiac and Pierron [35]. Here the method is extended to large deformation, using the finite deformation theory to compute displacement and strain; besides the identification is performed neglecting the elastic part of the deformation. For another application of the VFM at large deformation see Guélon et al. [53].

\subsection{The principle of virtual power for finite} deformation

The principle of virtual power is an alternative way of expressing the equation of motion and equilibrium. Let us consider a material body $\mathscr{B}$ which is subjected to a deformation process in time into an Euclidean space. The space occupied by the body at the initial instant $t_{0}$ is called the reference placement and indicated by $\mathscr{B}_{0}$ while the position at a time $t$ is called the current placement and is indicated by $\mathscr{B}_{t}$, see Fig. 8 . Using a cartesian coordinate system an arbitrary material point in the reference placement is denoted by the position vector $\mathbf{x}_{0}$ and the same point in the current placement is denoted by the position vector $\mathbf{x}$. It is possible to pass from one configuration to the other by the motion function $\chi$. The field of displacement vectors is the difference of the position vectors of a material point in the current and in the reference placement.

$\mathbf{u}\left(\mathbf{x}_{0}, t\right)=\chi\left(\mathbf{x}_{0}, t\right)-\mathbf{x}_{0}$

All the local deformation measures are obtained from the deformation gradient $\mathbf{F}$ defined as: 


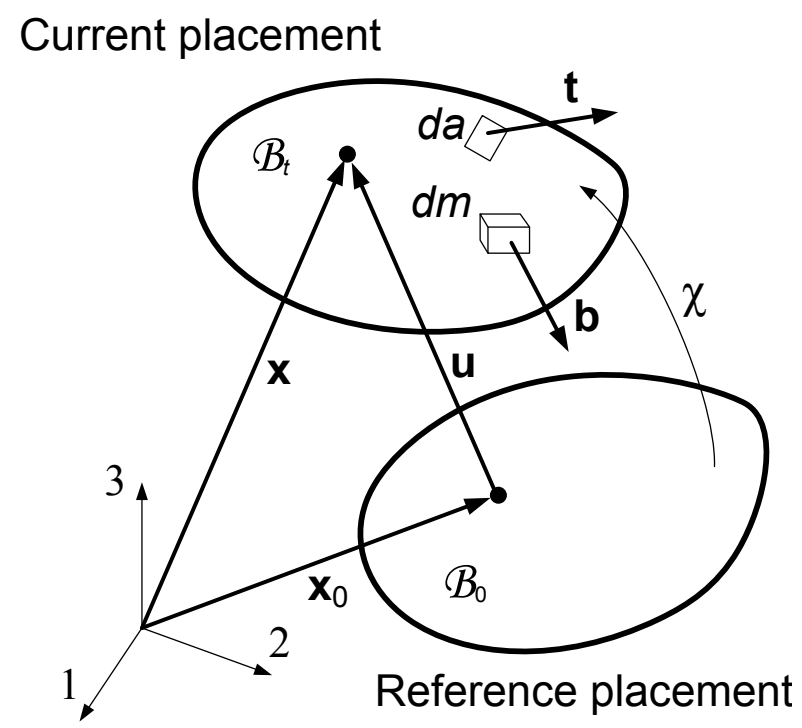

Fig. 8 Description of the motion of a material body, reference placement and current placement at the time $t$. The body in the current placement is subjected to surface loads and body forces.

$\mathbf{F}=\operatorname{Grad} \boldsymbol{\chi}\left(\mathbf{x}_{0}, t\right)=\operatorname{Grad} \mathbf{u}\left(\mathbf{x}_{0}, t\right)+\mathbf{I}$

$\mathbf{F}$ is dimensionless and assumed to be invertible at each point and each time. The polar decomposition allows to separate the deformation and the rotation parts of $\mathbf{F}$ :

$\mathbf{F}=\mathbf{R U}=\mathbf{V R}$

where $\mathbf{R}$ is the rotation tensor and $\mathbf{U}$ and $\mathbf{V}$ are the right and left stretch tensors. At time $t$ the body $\mathscr{B}_{t}$ is subjected to a general distribution of surface loads $\mathbf{t}$ and body forces $\mathbf{b}$.

Let us now consider an arbitrary kinematically admissible vector field $\delta \mathbf{v}$, called virtual velocity, as test function. The principle of virtual power states:

$$
\begin{aligned}
\int_{\mathscr{B}_{t}} \mathbf{T} \cdot \delta \mathbf{D} d v+ & \int_{\mathscr{B}_{t}} \mathbf{a} \cdot \delta \mathbf{v} d m= \\
& \int_{\partial \mathscr{B}_{t}}(\mathbf{T n}) \cdot \delta \mathbf{v} d a+\int_{\mathscr{B}_{t}} \mathbf{b} \cdot \delta \mathbf{v} d m
\end{aligned}
$$

with

$\delta \mathbf{D}=\frac{1}{2}\left(\operatorname{grad} \delta \mathbf{v}+\operatorname{grad} d^{T} \delta \mathbf{v}\right)$

The term virtual power derives from the fact that $\delta \mathbf{v}$ can be seen as a virtual velocity field and $\delta \mathbf{D}$ as a virtual stretch rate, in this sense Eq. 7 becomes a balance between the virtual power of the internal and external forces.

The principle of virtual power is written in the current placement $\mathscr{B}_{t}$ using an Eulerean or spatial description. In this specific case, it is more advantageous to rewrite it in terms of the reference placement $\mathscr{B}_{0}$ using a Lagrangean or material description. Mathematically both descriptions are equivalent. It writes:

$$
\begin{aligned}
\int_{\mathscr{B}_{0}} \mathbf{T}^{1 P K} \cdot \delta \mathbf{F}^{\bullet} d v_{0}+\int_{\mathscr{B}_{0}} \mathbf{a} \cdot \delta \mathbf{v} d m_{0}= \\
\int_{\partial_{\mathscr{B}_{0}}}\left(\mathbf{T}^{1 P K} \mathbf{n}_{0}\right) \cdot \delta \mathbf{v} d a_{0}+\int_{\mathscr{B}_{0}} \mathbf{b} \cdot \delta \mathbf{v} d m_{0}
\end{aligned}
$$

where

$$
\delta \mathbf{F}^{\bullet}=\operatorname{Grad} \delta \mathbf{v}\left(\mathbf{x}_{0}, t\right)
$$

and $\mathbf{T}^{1 P K}$ is a different stress measurement, namely the 1st Piola-Kirchhoff stress tensor:

$\mathbf{T}^{1 P K}=\operatorname{det}(\mathbf{F}) \mathbf{T} \mathbf{F}^{-T}$

The 1st Piola-Kirchhoff stress tensor, also called engineering or nominal stress, relates forces applied in the current placement with areas in the reference placement [14]. All the quantities refer to the material points in the reference placement using the position vector $\mathbf{x}_{0}$. If the deformation process is quasi-static, the inertia forces can be ignored. Neglecting also the body forces such as the body weight, Eq. 9 becomes:

$\int_{\mathscr{B}_{0}} \mathbf{T}^{1 P K} \cdot \delta \mathbf{F}^{\bullet} d v_{0}=\int_{\partial \mathscr{B}_{0}}\left(\mathbf{T}^{1 P K} \mathbf{n}_{0}\right) \cdot \delta \mathbf{v} d a_{0}$

which is valid at any time and for any admissible virtual field.

\subsection{The Virtual Fields Method for non-linear problems}

The Virtual Fields Method has already been extended successfully to many cases of non-linear problems like rubber, elasto-plasticity, etc. Here, the aim is to identify the constitutive parameters of a plasticity model when the displacement field of the specimen and the loading conditions during the test are known. The procedure is applied to heterogeneous displacement fields where no direct relationships are available between stress and strain fields.

Let us consider a general plasticity model governed by $N_{c p}$ parameters and call $\boldsymbol{\xi}=\left\{X_{1}, X_{2}, \ldots, X_{N_{c p}}\right\}$ the 
vector of the constitutive parameters which is the unknown of the problem. If the parameters are correct, the actual stress field inside a specimen can be computed from the measured strain field. The surface forces acting at the boundary of the specimen can be related to the loads measured during the test. The following cost function is defined:

$$
\begin{aligned}
\psi(\boldsymbol{\xi}, \delta \mathbf{v}, t)=\mid \int_{\mathscr{B}_{0}} \mathbf{T}^{1 P K} \cdot \delta \mathbf{F}^{\bullet} d v_{0}- \\
\int_{\partial \mathscr{B}_{0}}\left(\mathbf{T}^{1 P K} \mathbf{n}_{0}\right) \cdot \delta \mathbf{v} d a_{0} \mid
\end{aligned}
$$

According to the principle of virtual power (see Eq. 12), the function $\psi(\boldsymbol{\xi}, \delta \mathbf{v}, t)$ is zero for any admissible virtual fields $\delta \mathbf{v}$ and at any time $t$ of the test, when the parameters are correct. At this point a general cost function is assembled using $N_{v}$ admissible virtual fields and $N_{t}$ time step of the test. It writes:

$\Psi(\boldsymbol{\xi})=\sum_{i=1}^{N_{v}} \sum_{j=1}^{N_{t}} \psi\left(\boldsymbol{\xi}, \delta \mathbf{v}_{i}, t_{j}\right)$

The identification process consists in the minimization of the cost function with respect to the constitutive parameters. The final identified parameters are the ones that best verify the equilibrium law written in the form of the principle of virtual power.

In the following sections it will be shown how to compute the stress field from the measured displacement field and how to chose suitable virtual fields.

\section{Computation of the deviatoric stress field}

In a general plasticity model the following set of constitutive equations have to be defined: a yield criterion which indicates if the material undergoes plastic deformation; a directional flow rule which designates the direction of the plastic flow according to the stress state; a hardening rule which describes how the yield locus evolves because of the deformation process.

The proposed approach is limited to pressure-independent plasticity models, in this case the yield criterion $\Phi_{p}$ can be written as a function of the deviatoric stress tensor $\mathbf{S}$ :

$$
\Phi_{p}(\mathbf{S})=\sigma_{T}(\mathbf{S})-\sigma_{Y}=0
$$

where $\sigma_{T}$ is the equivalent stress and $\sigma_{Y}$ is the yield stress which is identified as the yield limit in a tensile test in certain material direction. In Fig. 9 a general yield locus is represented in the so called HaighWestergaard plane or principal deviator stress plane. $\mathbf{S}$

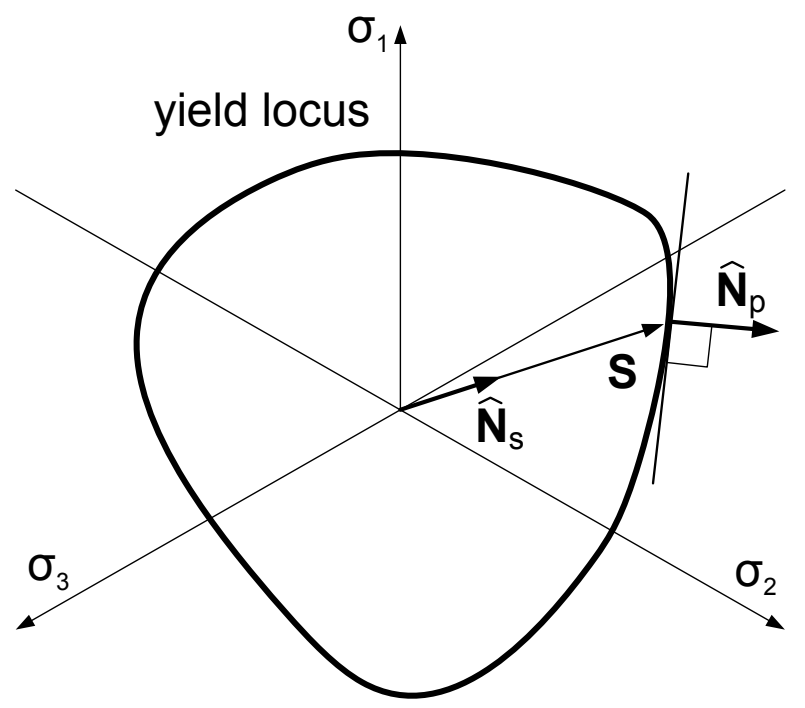

Fig. 9 Representation of a general yield locus in the HaighWestergaard plane. The normalised tensors $\widehat{\mathbf{N}}_{S}$ and $\widehat{\mathbf{N}}_{p}$ represent the direction of the deviatoric stress and the direction of the plastic flow respectively.

is a general deviatoric stress tensor which fullfils the yield condition and $\widehat{\mathbf{N}}_{S}$ is a normalised tensor such as:

$\mathbf{S}=|\mathbf{S}| \widehat{\mathbf{N}}_{S}$

The direction of the plastic flow, defined by the versor $\widehat{\mathbf{N}}_{p}$, is obtainable from the measured displacement field, therefore the aim is to find a function $\mathfrak{F}$ which returns the direction of the deviatoric stress versor from the plastic flow normal, that is:

$\widehat{\mathbf{N}}_{S}=\mathfrak{F}\left(\widehat{\mathbf{N}}_{p}\right)$

At this point the deviatoric stress tensor can be computed using Eq. 16 in Eq. 15 and solving the resultant equation for the scalar modulus $|\mathbf{S}|$ which is the only unknown.

$\sigma_{T}\left(|\mathbf{S}| \widehat{\mathbf{N}}_{S}\right)-\sigma_{Y}=0$

Using an associative flow rule the directions of the plastic flow is normal with respect to the yield surface at the yielding point, it follows:

$\widehat{\mathbf{N}}_{p}=\frac{\mathrm{d} \Phi_{p}(\mathbf{S})}{\mathrm{d} \mathbf{S}} /\left|\frac{\mathrm{d} \Phi_{p}(\mathbf{S})}{\mathrm{d} \mathbf{S}}\right|$

If the yield locus is convex and differentiable, the direction of the plastic flow is univocally determined by the direction of the deviatoric stress tensor and the 
function $\mathfrak{F}$ can be obtained inverting Eq. 19. The procedure is general and can be applied to any plasticity model when it is possible to find an invertible relation between the plastic flow direction and the deviatoric stress versor.

In the present case the procedure is applied to the Hill48 yield criterion given in Eq. 1. Denoting $\widehat{\mathbf{N}}_{p}=$ $\left\{\widehat{n}_{i j}^{p}\right\}$ and $\widehat{\mathbf{N}}_{S}=\left\{\widehat{n}_{i j}^{S}\right\}$, the numerator of Eq. 19 is computed as:

$\frac{\mathrm{d} \Phi_{p}(\mathbf{S})}{\mathrm{d} \mathbf{S}}=2|\mathbf{S}|\left[\begin{array}{cccccc}(h+g) & -h & -g & 0 & 0 & 0 \\ -h & (f+h) & -f & 0 & 0 & 0 \\ -g & -f & (g+f) & 0 & 0 & 0 \\ 0 & 0 & 0 & n & 0 & 0 \\ 0 & 0 & 0 & 0 & l & 0 \\ 0 & 0 & 0 & 0 & 0 & m\end{array}\right]\left\{\begin{array}{c}\widehat{n}_{11}^{S} \\ \widehat{n}_{22}^{S} \\ \widehat{n}_{33}^{S} \\ \widehat{n}_{12}^{S} \\ \widehat{n}_{23}^{S} \\ \widehat{n}_{31}^{S}\end{array}\right\}$

Since the matrix of Eq. 20 is singular, in order to find the inverse relation further conditions have to be introduced, viz:

$\operatorname{tr} \widehat{\mathbf{N}}_{p}=\operatorname{tr} \widehat{\mathbf{N}}_{S}=0$

where the trace of $\widehat{\mathbf{N}}_{p}$ is zero because of the volume conservation during the plastic flow and the trace of $\widehat{\mathbf{N}}_{S}$ is zero because the trace of the deviatoric stress is zero. After few mathematical steps the sought function $\mathfrak{F}$ can be expressed as the following linear transformation:

$\widehat{\mathbf{N}}_{S}=\frac{\mathbf{A} \widehat{\mathbf{N}}_{p}}{\left|\mathbf{A} \widehat{\mathbf{N}}_{p}\right|}$

where the numerator of Eq. 22 can be numerically computed as follows:

$\mathbf{A} \widehat{\mathbf{N}}_{p}=\left[\begin{array}{cccccc}\frac{2}{1+R} & -\frac{1}{1+R} & -\frac{R}{1+R} & 0 & 0 & 0 \\ -\frac{1}{1+R} & \frac{2}{1+R} & -\frac{R}{1+R} & 0 & 0 & 0 \\ -\frac{1}{1+R} & -\frac{1}{1+R} & \frac{2 R}{1+R} & 0 & 0 & 0 \\ 0 & 0 & 0 & \frac{1}{1+R} & 0 & 0 \\ 0 & 0 & 0 & 0 & \frac{2(1+2 R)}{3(1+R)^{2}} & 0 \\ 0 & 0 & 0 & 0 & 0 & \frac{2(1+2 R)}{3(1+R)^{2}}\end{array}\right]\left\{\begin{array}{c}\widehat{n}_{11}^{P} \\ \widehat{n}_{22}^{P} \\ \widehat{n}_{33}^{P} \\ \widehat{n}_{12}^{P} \\ \widehat{n}_{23}^{P} \\ \widehat{n}_{31}^{P}\end{array}\right\}$

$\mathbf{A}$ is a 4th-order tensor and the anisotropic constants have been rewritten in terms of the parameter $R$ using Eq. 2. It may be noted that only six components are represented in Eq. 23 because of the symmetry of the tensor, however the nine components have to be used to compute the tensor modulus at the denominator of Eq. 22. Once the direction of the deviatoric stress is established, the modulus is computed solving Eq. 18 written for the adopted yield criterion, it results:

$$
\begin{aligned}
& |\mathbf{S}|=\sigma_{Y}\left[\frac{1}{1+R}\left(\widehat{n}_{22}^{S}-\widehat{n}_{33}^{S}\right)^{2}+\frac{1}{1+R}\left(\widehat{n}_{33}^{S}-\widehat{n}_{11}^{S}\right)^{2}+\right. \\
& \left.\frac{R}{1+R}\left(\widehat{n}_{11}^{S}-\widehat{n}_{22}^{S}\right)^{2}+\frac{3}{2} \widehat{n}_{23}^{S}{ }^{2}+\frac{3}{2} \widehat{n}_{31}^{S}{ }^{2}+\frac{1+2 R}{1+R} \widehat{n}_{12}^{S}{ }^{2}\right]^{-\frac{1}{2}}
\end{aligned}
$$

where the yield stress $\sigma_{Y}$ is updated for the current time step using the hardening law given in Eq. 3.

4.1 Computation of the plastic flow direction from the displacement field

The plastic flow direction has to be computed from the measured displacement field. As explained in Section 2.2 the displacement field has been discretized over a three-dimensional mesh using brick elements and linear shape functions. The stress computation will therefore be performed at the integration points of each element. Here a single integration point, placed at the element centroid, was adopted. In the FE theory this is referred to as reduced integration and it is often preferred in the case of brick elements to avoid the shear locking and volumetric locking effect [54].

The plastic strain rate at the integration point of element $k$ and at time step $t$ of the test can be computed as follows:

$\mathbf{E}_{k}^{p}=\frac{\partial \mathbf{E}_{k}^{p}}{\partial t} \approx \frac{\mathbf{E}_{k}^{p(t)}-\mathbf{E}_{k}^{p(t-1)}}{\Delta t}=\frac{\Delta \mathbf{E}_{k}^{p(t)}}{\Delta t}$

such relation is valid if the strain increment $\Delta \mathbf{E}_{k}^{p(t)}$ is sufficiently small. Since the plastic deformation is isochoric in pressure-independent plasticity and, at large strains, the elastic part is small compared to the plastic one, the plastic strain tensor $\mathbf{E}_{k}^{p(t)}$ can be approximated to the deviatoric part of the total strain tensor $\mathbf{E}_{k}^{(t)}$, viz:

$\mathbf{E}_{k}^{p} \approx \mathbf{E}_{k}-\frac{1}{3} \operatorname{tr}\left(\mathbf{E}_{k}\right) \mathbf{I}$

The total strain tensor is computed as the spatial logarithmic strain or Henky strain tensor:

$\mathbf{E}_{k}=\ln \mathbf{V}_{k}$

where the stretch tensor $\mathbf{V}_{k}$ for element $k$ is obtained from the deformation gradient $\mathbf{F}_{k}$ using Eq. 6. The deformation gradient at the integration point is obtained from the nodal displacement using the shape functions, i.e. in a matrix form: 
$\left[\mathbf{F}_{k}\right]=\left(\left[\mathbf{B}_{k}\left(\mathbf{x}_{0 k}^{N}\right)\right]\left[\mathbf{u}_{k}^{N}\right]\right)^{T}$

where $\left[\mathbf{u}_{k}^{N}\right]$ is an $8 \times 3$ matrix which contains the displacement of the 8 nodes of the element $k$ along the 3 directions and $\left[\mathbf{B}_{k}\right]$ is a $3 \times 8$ gradient matrix which is obtained from the nodal coordinates $\mathbf{x}_{0}{ }_{k}^{N}$ and the shape functions. More details about how to compute the displacement gradient at the integration point of an element can be found in every FE textbook, cf. Zienkiewicz and Taylor [55], for instance.

Dealing with anisotropic plasticity, the yield criterion is defined in the material coordinate system oriented according to the texture of the material. Let us assume that in the undeformed body the material coordinate system coincides with the global coordinate system. During the deformation process the material coordinate system can change its orientation because of body rotations. The plastic strain tensor defined in Eq. 26 has to be rotated into the material coordinate system using the rotation tensor $\mathbf{R}$ obtained from the polar decomposition, see Eq. 6, it follows:

$$
\left.\mathbf{E}_{k}^{p}\right|_{\text {mat }}=\mathbf{R}_{k}{ }^{T} \mathbf{E}_{k}^{p} \mathbf{R}_{k}
$$

The direction of the plastic flow to be used in the computation of the deviatoric stress is the direction of the plastic strain rate in the material coordinate system, therefore using Eq. 25 and Eq. 29, for element $k$ and time step $t$, it writes:

$\widehat{\mathbf{N}}_{p_{k}}{ }^{(t)}=\frac{\left.\Delta \mathbf{E}_{k}^{p}\right|_{m a t} ^{(t)}}{\left|\Delta \mathbf{E}_{k}^{p}\right|_{m a t}^{(t)} \mid}$

From $\widehat{\mathbf{N}}_{p_{k}}^{(t)}$ the normalised tensor $\widehat{\mathbf{N}}_{S}{ }_{k}^{(t)}$ is computed using Eq. 22 and Eq. 23. The deviatoric stress tensor in the material coordinate system can be computed as follows according to Eq. 16:

$$
\left.\mathbf{S}_{k}^{(t)}\right|_{m a t}=\left|\mathbf{S}_{k}^{(t)}\right| \frac{\mathbf{A} \widehat{\mathbf{N}}_{p_{k}}^{(t)}}{\left|\mathbf{A} \widehat{\mathbf{N}}_{p_{k}}^{(t)}\right|}
$$

where $\left|\mathbf{S}_{k}^{(t)}\right|$ is obtained from Eq. 24. The deviatoric stress tensor can be expressed in the global coordinate system using the rotation tensor $\mathbf{R}_{k}^{(t)}$, viz:

$\mathbf{S}_{k}^{(t)}=\left.\mathbf{R}_{k}^{(t)} \mathbf{S}_{k}^{(t)}\right|_{\text {mat }} \mathbf{R}_{k}^{(t)^{T}}$

\subsection{Determination of the plastic range}

The procedure works only if plastic deformation takes place. During a test, especially if there is a strain localization, it may happen that parts of the specimen are under plastic deformation and others are unloaded and come back into the elastic range. A procedure is needed to find out which parts of the measurement volume are in the elastic range and which are in the plastic range. The simplest way of doing this is considering under plastic deformation the elements in which the strain increment $\Delta \mathbf{E}_{k}^{(t)}$ exceeds a fixed strain threshold.

Here a finer criterion is provided which however assumes that the elastic properties of the material are known, for instance they can be identified from the same test using the standard VFM. At the first step of the test, the specimen is stress free and with no accumulated plastic deformation:

$\left\{\begin{array}{l}\mathbf{S}_{k}^{(0)}=0 \\ p_{k}^{(0)}=0\end{array} \quad \forall\right.$ element $k$

At the current step $t$, for each element $k$, a trial deviatoric stress is obtained assuming that the increment is in the elastic range:

$\mathbf{S}_{k}^{\text {trial }}{ }^{(t)}=\mathbf{S}_{k}^{(t-1)}+\frac{E}{(1+\nu)} \Delta \mathbf{E}_{k}^{(t)}$

where $\Delta \mathbf{E}_{k}{ }^{(t)}$ is the deviatoric strain tensor as in Eq. 26. All the stress and strain tensors are expressed in the material coordinate system although the subscript $\left.\right|_{\text {mat }}$ has been omitted for the sake of clarity. The yield criterion (Eq. 1) and the hardening law (Eq. 3) are then used to check if the element is in the elastic or plastic range. Two cases are possible:

1. the element is in the elastic range

$$
\begin{aligned}
& \sigma_{T}\left(\mathbf{S}_{k}^{\text {trial }}(t)\right)-\sigma_{Y}\left(p_{k}^{(t-1)}\right)<0 \\
& \text { then }\left\{\begin{array}{l}
S_{k}^{(t)}=\mathbf{S}_{k}^{\text {trial }}(t) \\
p_{k}^{(t)}=p_{k}^{(t-1)}
\end{array}\right.
\end{aligned}
$$

2. the element is in the plastic range

$$
\sigma_{T}\left(\mathbf{S}_{k}^{\text {trial }}(t)\right)-\sigma_{Y}\left(p_{k}^{(t-1)}\right)>0
$$

$$
\text { then }\left\{\begin{array}{l}
S_{k}^{(t)} \text { computed from Eq. } 31 \\
p_{k}^{(t)}=p_{k}^{(t-1)}+\sqrt{\frac{2}{3} \Delta \mathbf{E}_{k}^{p(t)} \cdot \Delta \mathbf{E}_{k}^{p(t)}}
\end{array}\right.
$$




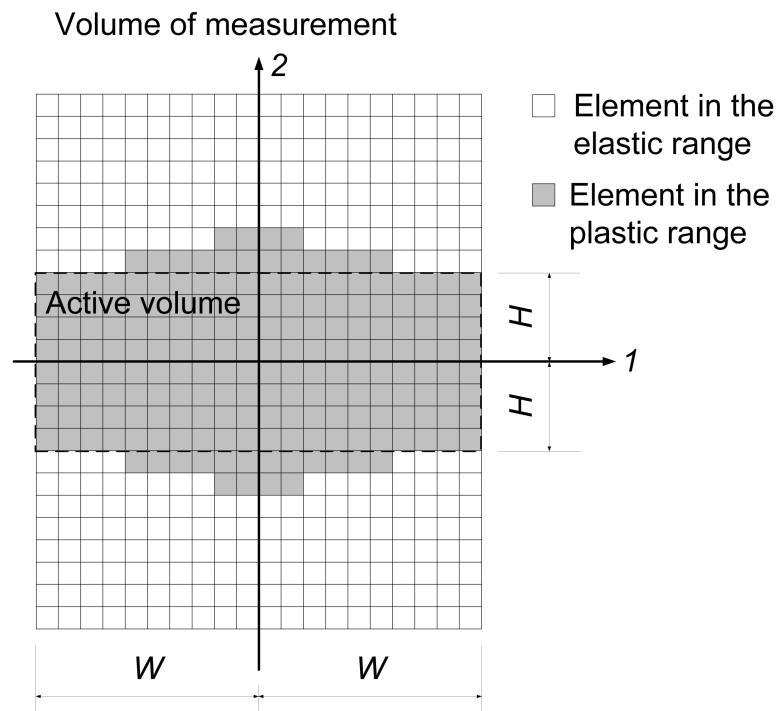

Fig. 10 Definition of the active volume. The computation of the cost function is restricted to the zone of the volume of measurement, called the active volume, in which all the elements are subjected to plastic deformation.

It is worth nothing that the procedure is valid both for loading and unloading. In order to study the initial part of the test, when the first yielding occurs, a further refinement could be done splitting the increments where the deformation changes from elastic to plastic [35]. Here such feature was not adopted because the aim of the study is to characterize the specimen behaviour at large strains, when the plastic flow is completely developed.

For each step of the test, a portion of the measurement volume is then flagged in which all the elements are subjected to plastic deformation, this portion is referred to hereafter as the active volume. The concept is explained in Fig. 10, the width of the active volume, $2 W$, is the same as the width of the volume of measurement and equals the total width of the specimen; the height of the active volume $2 H$ varies according to the elements that are in the plastic range. In Fig. 11 the evolution of the height $H$ during the test is plotted as a function of the reference time. After the yielding point, the whole measurement zone is subjected to plastic deformation, then, as the strain localizes at the centre, the height of the active volume decreases.

The idea here is that the zones of the specimen in the elastic range are not useful for the identification of the constitutive parameters of the plasticity model, indeed they can only be a source of undesired noise. The cost function will be evaluated only in the elements of the active volume.

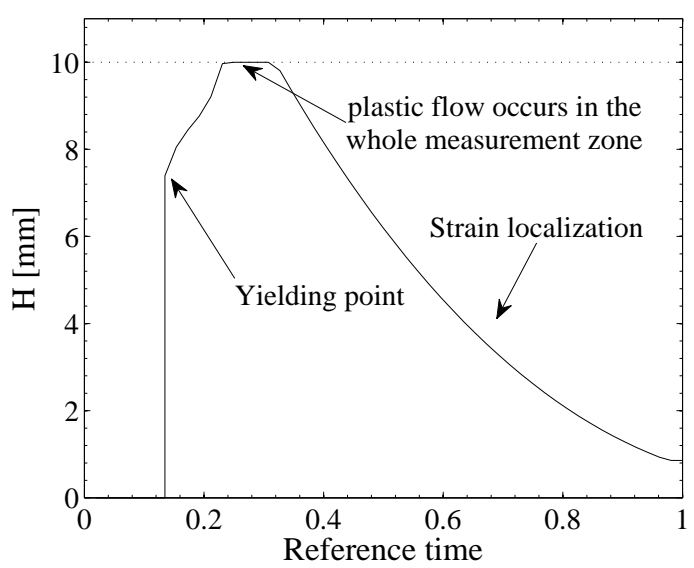

Fig. 11 Evolution of the height $H$ of the active volume. At the beginning the whole measurement area is subjected to plastic deformation $(H=10 \mathrm{~mm})$, then it decreases in order to keep into account only the elements subjected to plastic deformation.

\section{Choice of the virtual fields}

The cost function described by Eq. 13 is composed of two integrals, one over the volume $\mathscr{B}_{0}$ which represents the virtual power of the internal forces and the other over the surface of the body $\partial \mathscr{B}_{0}$ which represents the rate of virtual work of the external forces. According to the VFM the first integral is computed using the measured displacement fields and the second one is computed using the loads measured during the test.

A key point of the proposed identification procedure is the possibility of using the deviatoric stress in the computation of the virtual power of the internal forces, because, as explicated in the previous section, the deviatoric stress tensor can be computed from a general three dimensional displacement field without making any assumptions on the stress state. This can be done using suitable virtual fields.

5.1 Computation of the virtual power of the internal forces in terms of the deviatoric stress

The virtual power of the internal forces is computed using the 1st Piola-Kirchhoff stress tensor which is computed from the deformation gradient $\mathbf{F}$ and the Cauchy stress tensor T, see Eq. 11 and Eq. 12. The deformation gradient is obtained from the measured displacement field using Eq. 28, but, unfortunately, using the procedure presented in Section 4, it is possible to derive only the deviatoric part of the Cauchy stress tensor.

The problem is overcome by defining suitable virtual fields. Rewriting the volume integral of Eq. 13 using the definition of the 1st Piola Kirchhoff tensor given 
in Eq. 11 and the definition of deviatoric stress (i.e. $\left.\mathbf{S}=\mathbf{T}-\frac{1}{3} \operatorname{tr}(\mathbf{T}) \mathbf{I}\right)$, it follows:

$$
\begin{gathered}
\int_{\mathscr{B}_{0}} \mathbf{T}^{1 P K} \cdot \delta \mathbf{F}^{\bullet} d v_{0}=\int_{\mathscr{B}_{0}} \operatorname{det}(\mathbf{F}) \mathbf{T F}^{-T} \cdot \delta \mathbf{F}^{\bullet} d v_{0}= \\
\int_{\mathscr{B}_{0}} \operatorname{det}(\mathbf{F})\left(\mathbf{S}+\frac{1}{3} \operatorname{tr}(\mathbf{T}) \mathbf{I}\right) \mathbf{F}^{-T} \cdot \delta \mathbf{F}^{\bullet} d v_{0}= \\
\int_{\mathscr{B}_{0}} \operatorname{det}(\mathbf{F}) \mathbf{S ~ F}^{-T} \cdot \delta \mathbf{F}^{\bullet} d v_{0}+ \\
\int_{\mathscr{B}_{0}} \operatorname{det}(\mathbf{F}) \frac{1}{3} \operatorname{tr}(\mathbf{T}) \mathbf{F}^{-T} \cdot \delta \mathbf{F}^{\bullet} d v_{0}
\end{gathered}
$$

Hence, defining a virtual field $\delta \mathbf{v}$ such that:

$\mathbf{F}^{-T} \cdot \delta \mathbf{F}^{\bullet}\left(\delta \mathbf{v}\left(\mathbf{x}_{0}\right)\right)=0 \quad \forall \mathbf{x}_{0} \in \mathscr{B}_{0}$

the second integral in the last expression of Eq. 35 equals zero and the first one involves only the deviatoric stress. It follows that the virtual power of the internal forces is obtained directly from the deviatoric stress and the deformation gradient, viz:

$$
\int_{\mathscr{B}_{0}} \mathbf{T}^{1 P K} \cdot \delta \mathbf{F}^{\bullet} d v_{0}=\int_{\mathscr{B}_{0}} \operatorname{det}(\mathbf{F}) \mathbf{S} \mathbf{F}^{-T} \cdot \delta \mathbf{F}^{\bullet} d v_{0}
$$

It is interesting to compare the same condition written in terms of the current placement $\mathscr{B}_{t}$ using the Eulerean representation. The virtual power of the internal forces assumes this form (cf. Eq. 7):

$$
\begin{aligned}
\int_{\mathscr{B}_{0}} \mathbf{T}^{1 P K} \cdot \delta \mathbf{F}^{\bullet} d v_{0}= & \int_{\mathscr{B}_{t}} \mathbf{T} \cdot \delta \mathbf{D} d v= \\
& \int_{\mathscr{B}_{t}}\left(\mathbf{S}+\frac{1}{3} \operatorname{tr}(\mathbf{T}) \mathbf{I}\right) \cdot \delta \mathbf{D} d v
\end{aligned}
$$

The condition of Eq. 36 becomes:

$$
\mathbf{I} \cdot \delta \mathbf{D}(\delta \mathbf{v}(\mathbf{x}))=\operatorname{tr}(\delta \mathbf{D})=0 \quad \forall \mathbf{x} \in \mathscr{B}_{t}
$$

which means that the virtual velocity field has to satisfy virtual volume conservation. This intuitively suggests why the integral is independent from the hydrostatic stress which is the part of the stress tensor responsible for the volume change.
5.2 Computation of the virtual power of the external forces

The surface integral of Eq. 13 represents the action of the surface forces and can be regarded as the virtual power of the external forces. From an actual test it is easy to measure the resultants of the boundary forces using for instance load cells, but their distribution over the specimen is in general unknown. A way to overcome this problem with the VFM is to chose virtual fields which are constant in the parts of the body surface subjected to boundary forces. Let us consider a portion of the surface $\partial \mathscr{B}_{0}$, named $\partial \mathscr{S}_{0}$, where a boundary force distribution with resultant $\mathbf{f}$ is applied. By choosing a virtual field $\delta \mathbf{v}$ such that:

$\delta \mathbf{v}\left(\mathbf{x}_{0}\right)=\overline{\delta \mathbf{v}} \quad \forall \mathbf{x}_{0} \in \partial \mathscr{S}_{0}$

the constant value can be placed out of the integral:

$$
\int_{\partial \mathscr{S}_{0}}\left(\mathbf{T}^{1 P K} \mathbf{n}_{0}\right) \cdot \overline{\delta \mathbf{v}} d a_{0}=\left(\int_{\partial \mathscr{S}_{0}}\left(\mathbf{T}^{1 P K} \mathbf{n}_{0}\right) d a_{0}\right) \cdot \overline{\delta \mathbf{v}}
$$

According to the definition of the 1st Piola-Kirchhoff tensor, the integral under parenthesis can be rewritten in terms of the current placement and it equals the resultant of the surface forces, viz:

$$
\left(\int_{\partial \mathscr{S}_{0}}\left(\mathbf{T}^{1 P K} \mathbf{n}_{0}\right) d a_{0}\right) \cdot \overline{\delta \mathbf{v}}=\left(\int_{\partial \mathscr{S}} \mathbf{t} d a\right) \cdot \overline{\delta \mathbf{v}}=\mathbf{f} \cdot \overline{\delta \mathbf{v}}
$$

5.3 Definition of the virtual fields used in the identification procedure

The choice of the virtual fields plays an important role in the identification. Different techniques can be adopted, e.g. in recent applications of the VFM a procedure to automatically generate optimized virtual fields by a minimization of the sensitivity to noise was developed in Avril et al. [56] and adapted to elasto-plasticity in Pierron et al. [37]. In the present application noise is less of an issues than for elasticity, therefore the virtual fields have been manually defined, however other possibilities could be explored in the future.

The same mesh used to describe the displacement field is adopted to describe the virtual fields in the active volume in terms of virtual nodal velocity, see also Avril and Pierron [52]. Such approach is not mandatory, the virtual fields can be defined using polynomial 
functions or different mesh but it is convenient because it considerably simplifies the computations. The gradient of the virtual velocity field at each integration point can be defined as:

$$
\left[\delta \mathbf{F}_{k}^{\bullet}\right]=\left(\left[\mathbf{B}_{k}\right]\left[\delta \mathbf{v}_{k}^{N}\right]\right)^{T}
$$

where $\left[\delta \mathbf{v}_{k}^{N}\right]$ is an $8 \times 3$ matrix defining the virtual velocity of the 8 nodes of element $k$ and $\left[\mathbf{B}_{k}\right]$ is the matrix defined in Eq. 28. Although the same mesh and the same shape functions are employed, the nodal displacements $\left[\mathbf{u}_{k}^{N}\right]$ in Eq. 28 come from experimental (here, simulated) measurements while the virtual nodal velocity is an arbitrary function defined by the user.

In order to use the deviatoric stress in the computation of the cost function, the condition given in Eq. 36 has to be valid at each integration point, i.e.:

$\mathbf{F}_{k}^{-T}\left(\mathbf{u}_{k}^{N}\right) \cdot \delta \mathbf{F}_{k}^{\bullet}\left(\delta \mathbf{v}_{k}^{N}\right)=0 \quad \forall$ element $k$

where $\mathbf{u}_{k}^{N}$ is the measured displacement field at the element nodes and $\delta \mathbf{v}_{k}^{N}$ is the virtual velocity field at the element nodes. In the present application, in order to satisfy such condition, the virtual velocity field is defined only for the 1st and 2nd-directions, then, at each element, a programming routine adjusts the nodal velocity in the 3rd direction according to the value of the deformation gradient $\mathbf{F}_{k}$ in such a way as to satisfy Eq. 44, see the Appendix for more details.

Two virtual fields have been defined, namely $a$ and $b$ :

$$
\begin{gathered}
\delta \mathbf{v}_{a}=\left\{\begin{array}{l}
\delta v_{1}=0 \\
\delta v_{2}=\frac{x_{2}}{H} \\
\delta v_{3} \text { so that } \mathbf{F}_{k}^{-T} \cdot \delta \mathbf{F}_{k}^{\bullet}=0
\end{array}\right. \\
\delta \mathbf{v}_{b}=\left\{\begin{array}{l}
\delta v_{1}=\frac{x_{1}}{W} \frac{\left(\left|x_{2}\right|-H\right)}{H} \\
\delta v_{2}=0 \\
\delta v_{3} \text { so that } \mathbf{F}_{k}^{-T} \cdot \delta \mathbf{F}_{k}^{\bullet}=0
\end{array}\right.
\end{gathered}
$$

where $H$ and $W$ are the semi-height and the semiwidth of the active volume. The virtual velocity at the boundary nodes where the traction force is applied (i.e. where $\left.x_{2}= \pm H\right)$ is respectively $\overline{\delta \mathbf{v}_{a}}=\{0, \pm 1,0\}$ and $\overline{\delta \mathbf{v}_{b}}=\{0,0,0\}$. Consequently the rate of the external work, Eq. 42, returns the resultant of the vertical forces, i.e. the traction force $F$, for virtual field $a$ and zero for virtual field $b$. The two adopted virtual fields have been schematically represented in Fig. 12. The global cost function for the two virtual fields becomes:

$$
\begin{array}{r}
\Psi(\boldsymbol{\xi})=\sum_{t}^{N_{t}} \mid\left(\sum_{k}^{N_{E}} \operatorname{det} \mathbf{F}_{k}^{(t)} \mathbf{S}_{k}^{(t)} \mathbf{F}_{k}^{(t)}{ }^{-T} \cdot \delta \mathbf{F}_{a k}^{\bullet(t)} \Delta v_{0 k}\right) \\
-2 F^{(t)}|+| \sum_{k}^{N_{E}} \operatorname{det} \mathbf{F}_{k}^{(t)} \mathbf{S}_{k}^{(t)} \mathbf{F}_{k}^{(t)}{ }^{-T} \cdot \delta \mathbf{F}_{b k}^{\bullet(t)} \Delta v_{0 k} \mid
\end{array}
$$

where the integrals of Eq. 13 are approximated by a sum over the quantities computed at the integration point of each element. The deviatoric stress tensor $\mathbf{S}_{k}^{(t)}$ is expressed in the global coordinate system using Eq. 32.

\section{Results and discussion}

The procedure was implemented in MATLAB and used to identify the constitutive parameters of the simulated experiment. The displacement field and traction force obtained from the FE model have been used as input in the identification procedure. The identified parameters have then been compared to the ones input in the simulation.

The influence of the initial guess, the influence of the mesh used to describe the displacement field and an initial study about the influence of noise are presented here.

\subsection{Minimization of the cost function}

The whole identification procedure is summarized in the flow chart of Fig. 13. The input data are the specimen geometry, the 3D displacement field, the tensile force and the chosen constitutive model. As a first step the data are processed in order to obtain the deformation tensors and the virtual fields at each integration point and at each time step. Such operation has to be performed only once because the unknown constitutive parameters do not enter in the computations. After this data preprocessing, the parameters are identified finding the minimum of the cost function. The adopted minimization routine is the SQP algorithm [57] implemented in Matlab [58].

The stability of the procedure has been checked looking at the influence of the initial guess input in the minimization algorithm. Three different initial values have been used within the parameter application range. The results are listed in Table 3. The error of the identified parameters relatives to the hardening behaviour $\left(K_{H}, \varepsilon_{0}, N_{H}\right)$ is computed as the least square error between the equivalent stress-strain curve input in 

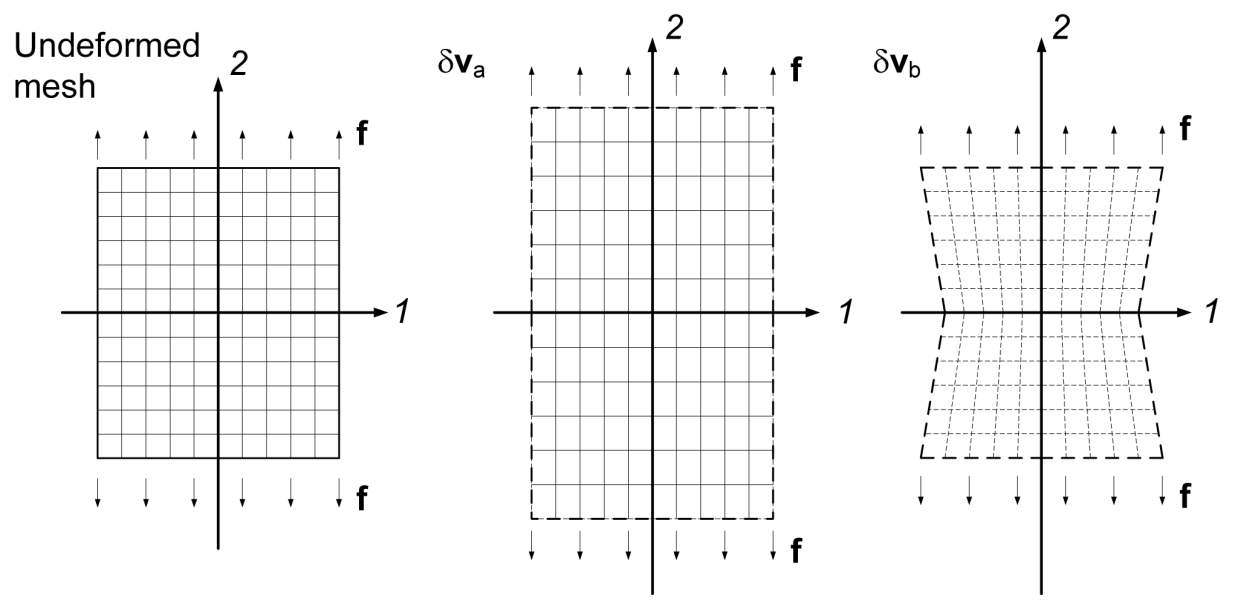

Fig. 12 Schematic view of the adopted virtual fields $\delta \mathbf{v}_{a}$ and $\delta \mathbf{v}_{b}$ illustrated as deformation of a regular mesh.
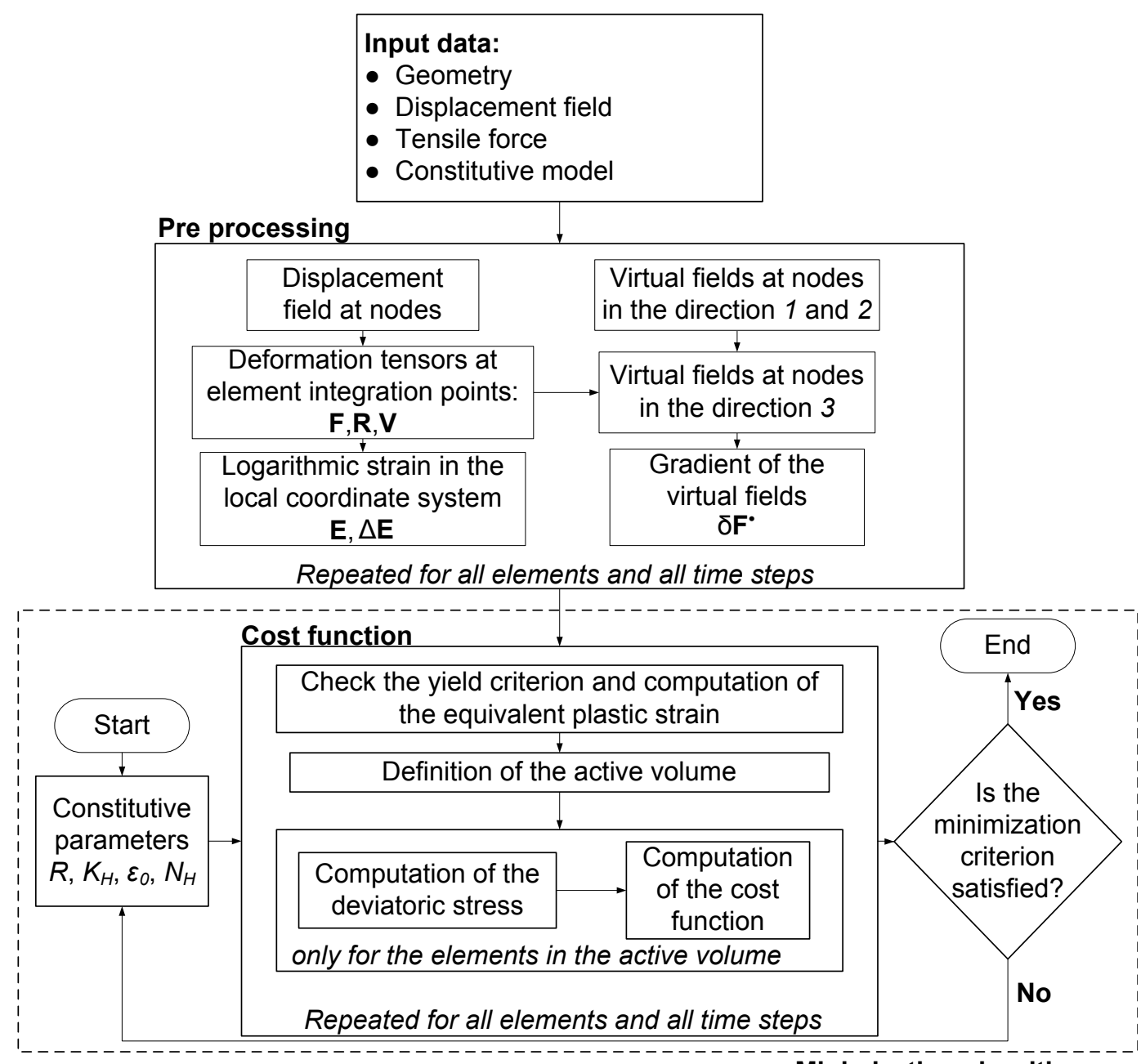

Minimization algorithm

Fig. 13 Flow chart of the computation algorithm employed to find the best set of constitutive parameters. 
the FEM model and the one calculated with the identified parameters. The single parameters are not compared directly, because, for instance, parameter $\varepsilon_{0}$ is not as influent as parameter $K_{H}$ in the description of the stress-strain curve.

When both virtual fields are used in the cost function, the parameters are correctly identified with errors less than $1 \%$. The solution is independent from the first guess. If only virtual field $\delta \mathbf{v}_{a}$ is used, the solution is still stable but the identification is not satisfactory, especially in the identification of the anisotropic parameter $R$. Using only virtual field $\delta \mathbf{v}_{b}$ in two cases is not possible to obtain a converged solution and in the third case it is possible to correctly identify only the parameter $R$. The choice of appropriate virtual fields is essential for a good identification.

An important remark has to be made about the computational time. For instance in the first case of Table 3 the cost function has to be computed 233 times to complete the minimization, using the proposed method the CPU time needed to evaluate the cost function, on a standard laptop (Intel Dual Core 2.27GHz, RAM 4GB), is around 2 seconds and the whole minimization procedure takes less than 10 minutes. If the same problem was handled with an FE updating technique, at each cost function evaluation an FE run would have to be launched. In this case the time required to perform the numerical simulation is around 300 seconds on the same laptop, that means that the time required to compute the cost function will be more than 100 times greater. This fact testifies the high potentiality of the proposed method in analyzing complex phenomena which cannot be described using simple two-dimensional models.

As explained in section 2.2, the displacement field has been extracted from the numerical model using four measurement grids. The effect of the adopted grid on the identification is illustrated in Table 4.

A coarser mesh reduces the quality of the identification since the displacement field is described in a more approximate way. Nevertheless, even using a very coarse mesh, the error remains under $5 \%$ in the identification of the stress-strain curve and under $10 \%$ in the identification of the Lankford parameter. Fig. 14 illustrates the stress-strain curves identified with the different measurement meshes. Using Mesh-3 only the first part of the stress-strain curve is correctly identified, then, as the stress localizes at the centre, the description of the displacement field becomes too rough to lead to a correct identification.

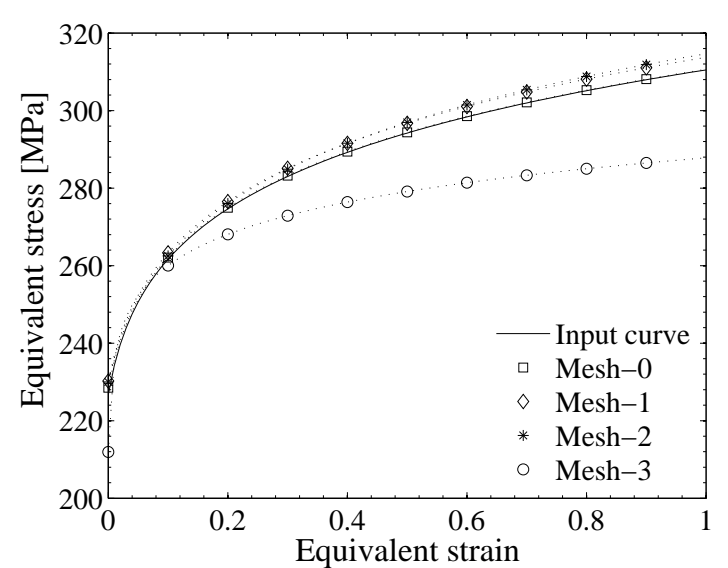

Fig. 14 Comparison of the stress-strain curves obtained with the parameter identified using different measurement meshes.

\subsection{Influence of noise}

The influence of noise and measurement errors in the identification from full-field measurements is a complex problem. It is beyond the aim of this paper to examine in details this aspect, however in this section a simple study is conducted to show how the proposed technique can handle noisy data.

At large strains the measurement of the total strain is not very sensitive to noise. However the noise can become a relevant problem in the computation of the strain rate where the strain increments $\Delta \mathbf{E}$, which can be rather small, have to be computed, see Eq. 25 .

A test was conducted adding a standard Gaussian white noise to the nodal displacement $\mathbf{u}^{N}$, before starting the identification procedure. The standard deviation of the input noise is $2 \mu \mathrm{m}$, this value is roughly the resolution of a commercial $2 \mathrm{D}$ DIC system with a field of view of $200 \times 200 \mathrm{~mm}$ (for instance see www.dantecdynamics.com).

In Fig. 15 a contour map of the strain increment component $\Delta E_{22}$ in the central section of the measurement volume is illustrated for the test time $t=0.7$, the presence of noise is clearly visible. To reduce the effect of noise a temporal smoothing can be performed by computing the strain rate at time $t$ using more time steps and performing a polynomial fitting. This can be efficiently done using a convolution method as the one provided by Savitzky and Golay [59].

In this case Eq. 25 can be rewritten as:

$\mathbf{E}_{k}^{p}=\frac{\partial \mathbf{E}_{k}^{p}}{\partial t} \approx \frac{\sum_{j=-m}^{m} h_{j} \mathbf{E}_{k}^{p(t+m)}}{\Delta t}$ 


\begin{tabular}{lcccccccc}
\hline & \multicolumn{9}{c}{ Hardening law } & \multicolumn{7}{c}{ Anisotropy } \\
& $K_{H}$ & $\varepsilon_{0}$ & $N_{H}$ & Err. & $R$ & Err. & Iter. & Eval. \\
\hline Reference & 310 & 0.02 & 0.08 & - & 0.6 & - & - & - \\
\hline First guess & 500 & 0.1 & 0.2 & - & 1.2 & - & - & - \\
Identified & 310.1 & 0.022 & 0.080 & $0.09 \%$ & 0.60 & $0.35 \%$ & 39 & 233 \\
only $\delta \mathbf{v}_{a}$ & 317.5 & 0.022 & 0.080 & $2.49 \%$ & 0.46 & $-22.9 \%$ & 49 & 315 \\
only $\delta \mathbf{v}_{b}$ & n.c. & n.c & n.c. & - & n.c. & - & 15 & 81 \\
\hline First guess & 150 & 0.01 & 0.05 & - & 1 & - & - & - \\
Identified & 310.1 & 0.022 & 0.080 & $0.09 \%$ & 0.60 & $0.35 \%$ & 30 & 188 \\
only $\delta \mathbf{v}_{a}$ & 317.5 & 0.022 & 0.080 & $2.49 \%$ & 0.46 & $-22.9 \%$ & 48 & 402 \\
only $\delta \mathbf{v}_{b}$ & n.c & n.c. & n.c. & - & n.c. & - & 4 & 26 \\
\hline First guess & 600 & 0.05 & 0.01 & - & 0.8 & - & - & - \\
Identified & 310.1 & 0.022 & 0.080 & $0.09 \%$ & 0.60 & $0.35 \%$ & 38 & 225 \\
only $\delta \mathbf{v}_{a}$ & 317.5 & 0.022 & 0.080 & $2.49 \%$ & 0.46 & $-22.9 \%$ & 40 & 266 \\
only $\delta \mathbf{v}_{b}$ & 600.0 & 0.199 & 0.144 & $94.8 \%$ & 0.59 & $-1.28 \%$ & 33 & 384 \\
\hline$*$ n.c. $=$ not converged & & & & & & &
\end{tabular}

Table 3 The robustness of the identification procedure is tested using different initial guesses chosen in the range of variabilities of the parameters. The identified parameters have been obtained using the two virtual fields described in Eq. 45 . The identification was then performed again using only virtual field $\delta \mathbf{v}_{a}$ or $\delta \mathbf{v}_{b}$ in the cost function. In the last two columns the number of iterations and the number of function evaluations are listed.

\begin{tabular}{lcccccc}
\hline \multirow{2}{*}{ Used mesh } & \multicolumn{3}{c}{ Hardening law } & \multicolumn{3}{c}{ Anisotropy } \\
& $K_{H}$ & $\varepsilon_{0}$ & $N_{H}$ & Err. & $R$ & Err. \\
\hline Mesh-0 & 310.1 & 0.022 & 0.080 & $0.09 \%$ & 0.602 & $0.35 \%$ \\
Mesh-1 & 313.0 & 0.025 & 0.083 & $0.86 \%$ & 0.602 & $0.53 \%$ \\
Mesh-2 & 313.9 & 0.028 & 0.087 & $0.89 \%$ & 0.62 & $2.60 \%$ \\
Mesh-3 & 287.8 & 0.001 & 0.044 & $4.62 \%$ & 0.64 & $7.40 \%$ \\
\hline Reference & 310 & 0.02 & 0.08 & - & 0.6 & - \\
First guess & 500 & 0.1 & 0.2 & - & 1.2 & - \\
\hline
\end{tabular}

Table 4 Dependence of identification on the 3D mesh used to regularize the measured displacement data.

$$
\text { Time }=0.70
$$

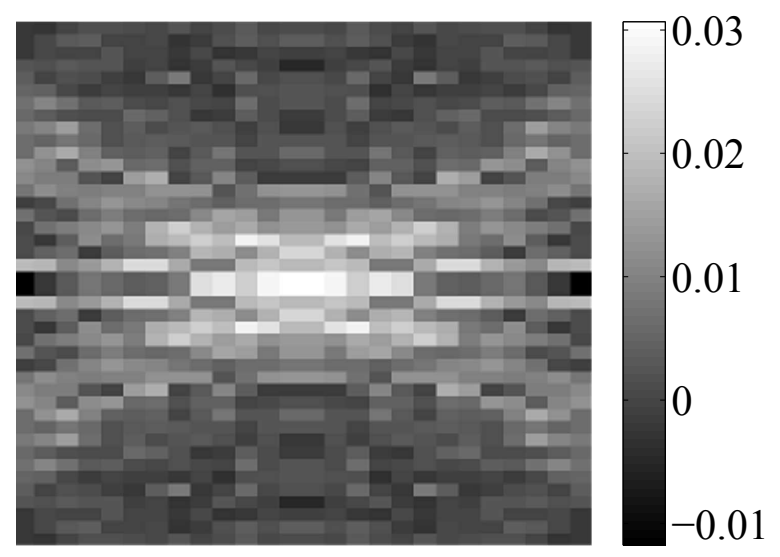

Fig. 15 Contour plot of the strain increment component $\Delta E_{22}$ at the test time $t=0.70$ in the central section of the volume of measurement. The effect of the introduced noise is clearly visible.
The strain rate at the time $t$ is computed using $2 m+1$ steps. A procedure to compute the convolution weights $h_{j}$ is given in Gorry [60], in this paper the method is extended to consider also the end points of the data set. In Fig. 16, the same strain increment of Fig. 15 is computed using Eq. 47, considering 5 and 15 points respectively.

The strain maps are filtered and the effect of the noise is almost zeroed using 15 points in the strain rate computation. The results in terms of identification are illustrated in Table 5. The identification error considerably decreases as long as more points are used to compute the strain rate. This feature can be very useful dealing with actual measurement data often affected by high level of noise, especially in case of three-dimensional full-field measurements.

Of course this is only a preliminary study to show the potentialities of the procedure, future in-depth analyses will have to be dedicated to this issue. 


\begin{tabular}{lcccccc}
\hline & \multicolumn{3}{c}{ Hardening law } & \multicolumn{3}{c}{ Anisotropy } \\
& $K_{H}$ & $\varepsilon_{0}$ & $N_{H}$ & Err. & $R$ & Err. \\
\hline Noisy data & 305.06 & 0.200 & 0.010 & $5.93 \%$ & 0.645 & $7.5 \%$ \\
Convolution (5 pts.) & 311.77 & 0.123 & 0.102 & $1.36 \%$ & 0.604 & $0.83 \%$ \\
Convolution (15 pts.) & 307.71 & 0.018 & 0.076 & $0.45 \%$ & 0.598 & $-0.33 \%$ \\
\hline Reference & 310 & 0.02 & 0.08 & - & 0.6 & - \\
First guess & 500 & 0.1 & 0.2 & - & 1.2 & - \\
\hline
\end{tabular}

Table 5 Effect of the noise in the identification. First the parameters have been identified using the noisy data, then the strain increment $\Delta \mathbf{E}$ has been computed using the convolution method with 5 and 15 points respectively.

\section{Differentiation over 5 steps}

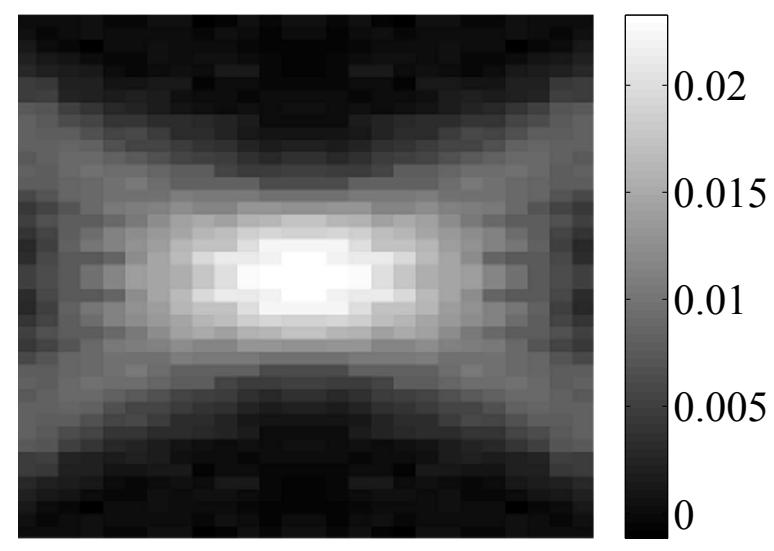

Differentiation over 15 steps

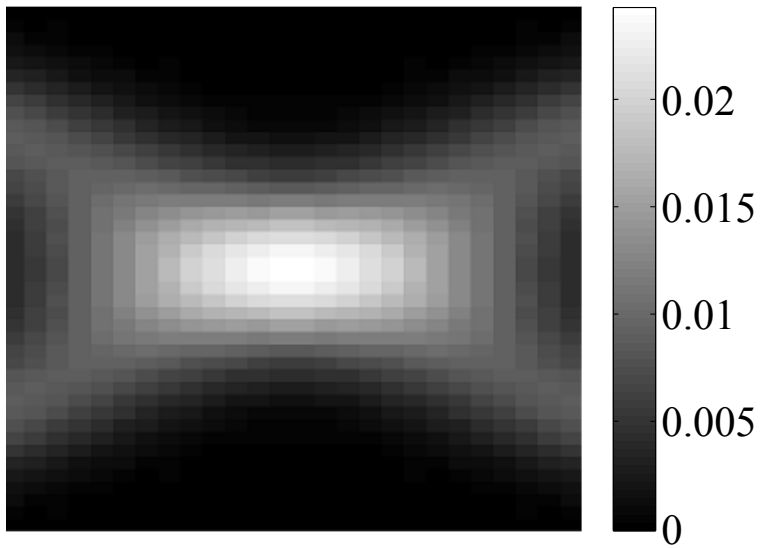

Fig. 16 Contour plot of the strain increment component $\Delta E_{22}$ at the test time $t=0.70$ obtained using the convolutions method. In the left plot 5 time steps have been used to compute the strain increment, in the right plot 15 time steps.

\section{Conclusions}

In this paper a procedure to extract the constitutive parameters of a plasticity model from a three dimensional displacement field is presented. The procedure is an application of the VFM at large deformations and it is valid for a general plasticity model although here it was implemented for the Hill48 anisotropic model. The main intent of the paper is to give a general description of the theory while its application to particular cases and to real experimental tests is left to upcoming papers, nevertheless an application example is illustrated using simulated experiments.

The procedure allows to directly utilize the full-field displacement data and the force measured during an experimental test to identify the constitutive parameters by the minimization of a cost function. It can be easily implemented in a program and used to process experimental data.

The presented technique is an effective tool to study the plastic behaviour at large deformation especially when a three dimensional representation is needed to describe phenomena such of the occurrence of necking or other plastic instabilities.

A first validation was performed looking at the stability of the procedure and the influence of noisy data was also studied by input artificial noise in the simulated displacement data.

The main outcomes of the paper are:

- a procedure is developed which allows to use a general 3D displacement field to directly evaluate the constitutive parameters of a general plasticity model; no hypotheses such as plane stress or plane strain have to be used;

- the procedure is well suited to be used in optimization algorithms, using the deviatoric stress in the evaluation of the cost function, no iterations are required to obtain the stress field from the displacement field, this feature enables to considerably reduce the computational time; 
- the method seems to be effective to allow for noisy measurements; the stress computation is based on the evaluation of the total strain which is usually rather large, besides a convolution derivative over multiple time increments can be used as a smoothing filter;

- the proposed implementation of the procedure requires that the volume measurement data are regularized in a 3D mesh, however the identification procedure is quite stable with respect to the size of the chosen discretization mesh.

In future works, the presented technique has to be validated on real data, using different plasticity models and different experimental tests. Simulated experiment could be used as well to evaluate the influence of the noise and establish what is the optimal configuration in terms of time increment and regularization grid size. Finally the use of automatically generated virtual fields can be introduced in the identification algorithm.

\section{Appendix}

As stated in section 5.3, the virtual velocity in the 3rd direction is computed using a programming routine in order to satisfy Eq. 44, in this appendix more details are given on how this routine is built up. For an arbitrary element $k$ the virtual velocities of the eight nodes are defined by the $8 \times 3$ matrix $\left[\delta \mathbf{v}_{k}^{N}\right]$, with:

$$
\left[\delta \mathbf{v}_{k}^{N}\right]=\left[\begin{array}{lll}
\delta v_{1}^{(1)} & \delta v_{2}^{(1)} & \delta v_{3}^{(1)} \\
\delta v_{1}^{(2)} & \delta v_{2}^{(2)} & \delta v_{3}^{(2)} \\
\delta v_{1}^{(3)} & \delta v_{2}^{(3)} & \delta v_{3}^{(3)} \\
\delta v_{1}^{(4)} & \delta v_{2}^{(4)} & \delta v_{3}^{(4)} \\
\delta v_{1}^{(5)} & \delta v_{2}^{(5)} & \delta v_{3}^{(5)} \\
\delta v_{1}^{(6)} & \delta v_{2}^{(6)} & \delta v_{3}^{(6)} \\
\delta v_{1}^{(7)} & \delta v_{2}^{(7)} & \delta v_{3}^{(7)} \\
\delta v_{1}^{(8)} & \delta v_{2}^{(8)} & \delta v_{3}^{(8)}
\end{array}\right]
$$

the numerical values in the first two columns of the matrix are evaluated using the nodal coordinates and the analytical description of the virtual fields given in Eq. 45. The values in third column, which represent the virtual velocity in the third direction, are computed using the routine. The routine is applied to all elements, at each iteration the following steps are repeated:

1. since the same node can be shared by several elements, the routine finds out which nodes of the element $k$ have an already assigned velocity $\delta v_{3}$, such nodes are excluded from the subsequent steps;
2. a constant displacement $\overline{\delta v_{3}}$ is assigned to the available nodes, the sign of $\overline{\delta v_{3}}$ is positive for the nodes in the lower face of the element and negative for the upper face.

3. the numerical value of $\overline{\delta v_{3}}$ is computed by solving the following equation:

$$
\left[\mathbf{F}_{k}^{-T}\right] \cdot\left(\left[\mathbf{B}_{k}\right]\left[\delta \mathbf{v}_{k}^{N}\left(\overline{\delta v_{3}}\right)\right]\right)^{T}=0
$$

which is the condition of Eq. 44 written using Eq. 43 to express $\delta \mathbf{F}_{k}^{*}$.

At the end of the procedure the virtual velocity is assigned to all nodes. The CPU time required to complete this operation is around 350 seconds which is comparable to the time needed to perform a numerical simulation of the test. However, as explained in section 6.1 and illustrated in the flow chart of Fig. 13, the routine has to be executed only once during the data preprocessing and does not enter in the computation of the cost function.

\section{References}

1. Hill R (1952) On discontinuous plastic states, with special reference to localized necking in thin sheets. J Mech Phys Solids 1(1):19-30

2. Nichols FA (1980) Plastic instabilities and uniaxial tensile ductilities. Acta Metall 28:663-673

3. Tvergaard N (1993) Necking in tensile bars with rectangular cross-section. Comp Meth Appl Mech Engrg 103:273-290

4. Cazacu O, Plunkett B, Barlat F (2006) Orthotropic yield criterion for hexagonal closed packed metals. Int J Plasticity $22: 1171-1194$

5. Plunkett B, Lebenson RA, Cazacu O, Barlat F (2006) Anisotropic yield function of hexagonal materials taking into account texture development and anisotropic hardening. Acta Mater 54:4159-4169

6. Barlat F, Yoon JW, Cazacu O (2007) On linear transformations of stress tensors for the description of plastic anisotropy. Int J Plasticity 23:876-896

7. Barlat F, Lege D, Brem J (1991) A six-component yield function for anisotropic materials. Int J Plasticity $7(7): 693-712$

8. Karafillis AP, Boyce MC (1993) A general anisotropic yield criterion using bounds and a transformation weighting tensor. J Mech Phys Solids 41:1859-1886

9. Darrieulat M, Piot D (1996) A method of generating analytical yield surfaces of crystalline materials. Int J Plasticity 12:575-610

10. Feigenbaum HP, Dafalias YF (2007) Directional distortional hardening in metal plasticity within thermodynamics. Int J Solids Struct 44:7526-7542

11. Voyiadjis GZ, Thiagarajan G, Petrakis E (1995) Constitutive modelling for granular media using an anisotropic distortional yield model. Acta Mech 110:151-171

12. Vegter H, van den Boogaard AH (2006) A plane stress yield function for anisotropic sheet material by interpolation of biaxial stress states. Int J Plasticity 22:557-580 
13. Bai Y, Wierzbicki T (2008) A new model of metal plasticity and fracture with pressure and Lode dependence. Int J Plasticity 24(6):1071-1096

14. Bertram A (2008) Elasticity and plasticity of large deofrmations, an introduction. Springer-Verlag Berlin Heidelberg

15. Hwang YM, Lin YK, Altan T (2007) Evaluation of tubular materials by a hydraulic bulge test. Int J Mach Tools Manuf 47:343-351

16. Hwang Y, Wang C (2009) Flow stress evaluation of zinc copper and carbon steel tubes by hydraulic bulge tests considering their anisotropy. J Mater Process Tech 209:4423-4428

17. Brunet M, Morestin F, Godereaux S (2001) Nonlinear kinematic hardening identification for anisotropic sheet metals with bending-unbending tests. J Eng Mater Technol 123:378-383

18. Laws V (1981) Derivation of the tensile stress-strain curve from bending data. J Mater Sci 16:1299-1304

19. Losilla G, Tourabi A (2004) Hardening of a rolled sheet submitted to radial and complex biaxial tensile loadings. Int J Plasticity 20:1789-1816

20. Mohr D, Oswald M (2008) A new experimental technique for the multi-axial testing of advanced high strength steel sheets. Exp Mech 48(1):65-77

21. Wierzbicki T, Bao Y, Lee Y, Bai Y (2005) Calibration and evaluation of seven fracture models. Int J Mech Sci 47(4-5):719-743

22. Cooreman S, Lecompte D, Sol H, Vantomme J, Debruyne C (2007) Elasto-plastic material parameter identification by inverse methods: calculation of the sensitivity matrix. Int J Solids Struct 44:4329-4341

23. Kajberg J, Lindkvist G (2004) Characterisation of materials subjected to large strains by inverse modelling based on in-plane displacement fields. Int J Solids Struct 41:3439-3459

24. Mahnken R, Stein E (1994) The identification of parameters for visco-plastic models via finite-element methods and gradient methods. Modelling Simul Mater Sci Eng 2:597-616

25. Meuwissen MHH, Oomens CWJ, Baaijens FPT, Petterson R, Janssen JD (1998) Determination of the elastoplastic properties of aluminium using a mixed numericalexperimental method. J Mater Process Tech 75:204-211

26. Tao H, Zhang N, Tong W (2009) An iterative procedure for determining effective stress-strain curves of sheet metals. Int J Mech Mater Des 5:13-27

27. Ling Y (1996) Uniaxial true stress-strain after necking. AMP J Tech 5:37-48

28. Zhang KS, Li ZH (1994) Numerical analysis of the stressstrain curve and fracture initiation for ductile material. Eng Fract Mech 49:235-241

29. Rossi M, Broggiato GB, Papalini S (2008) Application of digital image correlation to the study of planar anisotropy of sheet metals at large strains. Meccanica 43(2):185-199

30. Latourte F, Chrysochoos A, Pagano S, Wattrisse B (2008) Elastoplastic behavior identification for heterogeneous loadings and materials. Exp Mech 48:435-449

31. Romano AJ, Shirron JJ, Bucaro JA (1998) On the noninvasive determination of material parameters from a knowledge of elastic displacements: theory and numerical simulation. IEEE t ultrason ferr 45:751-759

32. Romano AJ, Bucaro JA, Ehman RL, Shirron JJ (2000) Evaluation of a material parameter extraction algorithm using MRI-based displacement measurements. IEEE t ultrason ferr 47:1575-1581
33. Avril S, Bonnet M, Bretelle A-S, Grédiac M, Hild F, Ienny P, Latourte F, Lemosse D, Pagano S, Pagnacco E, Pierron F (2008) Overview of identification methods of mechanical parameters based on full-field measurements. Exp Mech 48:381-402

34. Grédiac M, Pierron F, Avril S, Toussaint E (2006) The virtual fields method for extracting constitutive parameters from full-field measurements: a review. Strain 42:233-253

35. Grédiac M, Pierron F (2006) Applying the virtual fields method to the identification of elasto-plastic constitutive parameters. Int J Plasticity 22:602-627

36. Avril S, Pierron F, Pannier Y, Rotinat R (2008) Stress reconstruction and constitutive parameter identification in plane-stress elasto-plastic problems using surface measurements of deformation fields. Exp Mech 48:403-419

37. Pierron F, Avril S, The Tran V (2010) Extension of the virtual fields method to elasto-plastic material identification with cyclic loads and kinematic hardening. Int J Solids Struct 47:2993-3010

38. Pierron F, M A Sutton MA, Tiwari V (2010) Ultra high speed DIC and virtual fields method analysis of a three point bending impact test on an aluminium bar. Exp Mech. doi: 10.1007/s11,340-010-9402-y

39. Avril S, Pierron F, Sutton M, Yan J (2008) Identification of elasto-visco-plastic parameters and characterization of lüders behavior using digital image correlation and the virtual fields method. Mech Mater 40:729-742

40. Bay BK, Smith TS, Fyhrie DP, Saad M (1999) Digital volume correlation: Three-dimensional strain mapping using X-ray tomography. Exp Mech 39:217-226

41. Smith TS, Bay BK, Rashid MM (2002) Digital volume correlation including rotational degrees of freedom during minimization. Exp Mech 42:272.278

42. Verhulp E, van Rietbergen B, Huiskes R (2004) A threedimensional digital image correlation technique for strain measurements in microstructures. J Biomech 37:13131320

43. Gates M, Lambros J, Heath MT (2010) Towards high performance digital volume correlation. Exp Mech. doi: $10.1007 / \mathrm{s} 11,340-010-9445-0$

44. Franck C, Hong S, Maskarinec S, Tirrel D, Ravichandran G (2007) Three-dimensional full-field measurements of large deformations in soft materials using confocal microscopy and digital volume correlation. Exp Mech 43:207-218

45. Germaneau A, Doumalin P, Dupré J (2007) 3D strain field measurement by correlation of volume images using scattered light: recording of images and choice of marks. Strain 43:207-218

46. Roux S, Hild F, Viot P, Bernard D (2008) Threedimensional image correlation from X-ray computed tomography of solid foam. Compos Part A-Appl S 39:12351265

47. Rossi M, Chiappini G, Sasso M (2010) Characterization of aluminum alloys using a 3D full field measurement. In: 2010 SEM Annual Concerence \& Exposition on Experimental \& Applied Mechanics

48. Atay S, Kroenke C, Sabet A, Bayly P (2008) Measurement of the dynamic shear modulus of mouse brain tissue in-vivo by magnetic resonance elastography. J Biomech Eng 130:021013 (11p.)

49. Hill R (1948) A theory of the yielding and plastic flow of anisotropic metals. Proceedings of the Royal Society of London Series A, Mathematical and Physical Sciences 193:281-297 
50. Lankford WT, Snyder SC, Bausher JA (1950) New criteria for predicting the press performance of deep drawing sheets. Trans Am Soc Metals 42:1197-1232

51. Banabic D, Bunge HJ, Pöhlandt, Tekkaya A (2000) Formability of metallic materials. Springer Berlin

52. Avril S, Pierron F (2007) General framework for the identification of constitutive parameters from full-field measurements in linear elasticity. Int J Solids Struct 44:49785002

53. Guélon T, Toussaint E, Le Cam JB, Promma N, Grédiac M (2009) A new characterisation method for rubber. Polym Test 28:715-723

54. Dhondt G (2004) The finite element method for threedimensional thermomechanical applications. John Wiley \& Sons

55. Zienkiewicz OC, Taylor R (2006) The finite element method. Butterworth-Heinemann

56. Avril S, Grédiac M, Pierron F (2004) Sensitivity of the virtual fields method to noisy data. Comput Mech 34(6):439-452

57. Fletcher R, (1987) Practical Methods of Optimization. John Wiley and Sons

58. Constrained Nonlinear Optimization Algorithm (2011). From http://www.mathworks.com/help/toolbox/optim

59. Savitzky A, Golay MJE (1964) Smoothing and differentiation of data by simplified least squares procedures. Anal Chem 36:1627-1639

60. Gorry A (1990) General least-squares smoothing and differentiation by the convolution (Savitzky-Golay) method. Anal Chem 62:570-573 